\title{
Locally symmetric connections on complex surfaces and some equations of Monge-Ampère type
}

\author{
MARIA RoBAszewsKa
}

\begin{abstract}
We study locally symmetric connections induced by transversal bundles on non-degenerate complex surfaces. Each of such surfaces together with its transversal bundle can be described locally by a solution to some partial differential equation of Monge-Ampère type.
\end{abstract}

\section{Introduction}

Let $M$ be an $n$-dimensional connected complex manifold and $f: M \rightarrow \mathbf{C}^{n+1}$ a holomorphic immersion. Let $\mathcal{N}$ be a $\mathcal{C}^{\infty}$ transversal bundle, that is,

$$
\mathcal{N}=\bigcup_{p \in M} \mathcal{N}_{p}
$$

where $\mathcal{N}_{p}$ is a complex vector subspace of $\mathbf{C}^{n+1} \cong \mathbf{R}^{2 n+2}$ such that $f_{*}\left(T_{p} M\right) \oplus \mathcal{N}_{p}=\mathbf{C}^{n+1}$. The $\mathcal{C}^{\infty}$ class means that for any $q \in M$ there exists a neighbourhood $U$ of $q$ such that $\left.\mathcal{N}\right|_{U}$ is spanned over $\mathbf{C}$ by a vector field $\xi$ on $\mathbf{C}^{n+1}$ defined along $f$ :

$$
M \supset U \ni p \mapsto \xi_{p} \in T_{f(p)} \mathbf{C}^{n+1} \cong \mathbf{C}^{n+1},
$$

which is of class $\mathcal{C}^{\infty}$ and not necessarily holomorphic.

The connection $\nabla$, the $\mathbf{C}$-bilinear symmetric affine fundamental form $h=h_{1}+i h_{2}$, the affine shape operator $S$ and the transversal connection form $\tau=\mu+i \nu$ which are induced on $U$ by $f$ and $\xi$ are defined by the following Gauss and Weingarten formulae:

$$
\begin{aligned}
D_{X} f_{*} Y & =f_{*} \nabla_{X} Y+h_{1}(X, Y) \xi+h_{2}(X, Y) J \xi \\
D_{X} \xi & =-f_{*} S X+\mu(X) \xi+\nu(X) J \xi
\end{aligned}
$$

(see, e.g., $[4,5])$. Here $D$ denotes the standard connection on $\mathbf{C}^{n+1}$. The manifold $M$ is regarded as a $2 n$-dimensional real manifold with the complex 
structure $J$. To simplify notation, we use the same letter $J$ for the complex structure in $\mathbf{C}^{n+1} \cong \mathbf{R}^{2 n+2}$. The identification of $\mathbf{C}^{k}$ with $\mathbf{R}^{2 k}$ is given by: $\left(z^{1}+i z^{2}, \ldots, z^{2 k-1}+i z^{2 k}\right) \mapsto\left(z^{1}, z^{2}, \ldots, z^{2 k-1}, z^{2 k}\right)$.

$\widetilde{h}, \widetilde{S}$ and $\widetilde{\tau}$ :

$$
\begin{gathered}
\widetilde{\nabla}_{X} Y=\nabla_{X} Y-\frac{1}{\varphi+i \vartheta} h(X, Y) Z, \\
\widetilde{h}=\frac{1}{\varphi+i \vartheta} h, \\
\widetilde{S} X=(\varphi+i \vartheta) S X-\nabla_{X} Z+\widetilde{\tau}(X) Z, \\
\widetilde{\tau}(X)=\frac{X(\varphi)+i X(\vartheta)}{\varphi+i \vartheta}+\frac{1}{\varphi+i \vartheta} h(X, Z)+\tau(X) .
\end{gathered}
$$

Let $\xi$ and $\widetilde{\xi}$ be two local sections of $\mathcal{N}$. Then we have $\widetilde{\xi}=(\varphi+i \vartheta) \xi$ and $Z=0$, therefore $\widetilde{\nabla}_{X} Y=\nabla_{X} Y$ for any $\mathcal{C}^{\infty}$ vector fields $X, Y$ on $U$. It follows that $\nabla$ depends on $\mathcal{N}$ only and can be defined on the whole of $M$. Moreover, the complex rank of affine fundamental form $h$ does not depend on the transversal bundle $\mathcal{N}$. We call it the type number of $f$. This type number is constant on a dense open subset $M^{\prime}$ of $M$ [6]. The immersion is called non-degenerate, if $h$ is non-degenerate $(\forall X \neq 0 \exists Y: h(X, Y) \neq 0)$.

The induced connection $\nabla$, he affine fundamental form $h$, the shape operator $S$ and the transversal connection form $\tau$ satisfy the following fundamental equations.

Gauss equation:

$$
R(X, Y) Z=h(Y, Z) S X-h(X, Z) S Y .
$$

Codazzi equation for $h$ :

$$
\left(\nabla_{X} h\right)(Y, Z)+\tau(X) h(Y, Z)=\left(\nabla_{Y} h\right)(X, Z)+\tau(Y) h(X, Z) .
$$

Codazzi equation for $S$ :

$$
\left(\nabla_{X} S\right)(Y)-\tau(X) S Y=\left(\nabla_{Y} S\right)(X)-\tau(Y) S X .
$$

Ricci equation:

$$
h(X, S Y)-h(S X, Y)=2 d \tau(X, Y) .
$$


If $f$ is non-degenerate, there exists some canonical transversal bundle the complex affine normal bundle - which is the bundle of affine normal complex lines. According to the definition given by F. Dillen, L. Vrancken and L. Verstraelen in [1] (see also [2]), the affine normal complex line of $M$ at $p$ is a complex line in $T_{f(p)} \mathbf{C}^{n+1}$ determined by the complex affine normal vector $\xi_{p}$. The complex affine normal vector field $\xi$ is a local vector field on $\mathbf{C}^{n+1}$ defined along $f$ satisfying the following two conditions:

$$
{ }^{\mathrm{C}} H_{\xi}=1 \quad \text { and } \quad \tau=0
$$

Here $\tau$ is a transversal connection form and

$$
{ }^{\mathbf{C}} H_{\xi}:=\left|\operatorname{det}\left[h\left(X_{k}, X_{l}\right)\right]_{k, l=1}^{n}\right|^{2}
$$

where $X_{1}, \ldots, X_{n}$ is a local complex basis of $T M$ such that

$$
\left|{ }^{\mathbf{C}} \omega\left(f_{*} X_{1}, \ldots, f_{*} X_{n}, \xi\right)\right|=1
$$

The symbol $\mathbf{C}_{\omega}$ denotes the complex volume form on $\mathbf{C}^{n+1}$ such that $\mathbf{C}_{\omega}\left(e_{1}, \ldots, e_{n+1}\right)=1$ for the standard basis $e_{1}, \ldots, e_{n+1}$ of $\mathbf{C}^{n+1}$. Actually, if

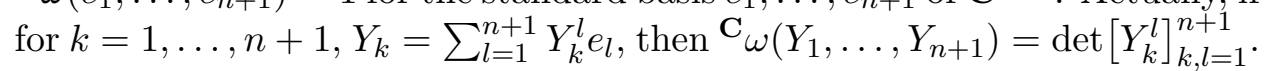

If $\xi$ and $\widetilde{\xi}$ are complex affine normal vector fields defined on the same open domain $U$, then there exists a real number $\theta$ such that $\widetilde{\xi}=e^{i \theta} \xi[1]$. It follows that for any $p \in M$ the affine normal complex line at $p$ is uniquely determined.

The aim of this paper is to give a local description of some of those immersions and transversal bundles for which the induced connection $\nabla$ is locally symmetric. The local symmetry of $\nabla$ is equivalent to the condition $\nabla R=0$, where $R$ is the curvature tensor of $\nabla$ [3]. Here we consider the case of a non-degenerate immersion, $\operatorname{dim}_{\mathbf{C}} M=2$ and we shall study connections of ranks 1 and 2. By the rank of locally symmetric connection we mean, following [8], the (complex) dimension of the subspace

$$
\operatorname{im} R_{x}:=\operatorname{span}_{\mathbf{R}}\left\{R(X, Y) Z: X, Y, Z \in T_{x} M\right\} .
$$

For any $x \in M,(1.11)$ is a complex subspace of $T_{x} M$. If $\nabla R=0$ and $M$ is connected, then $\operatorname{dim} \operatorname{im} R_{x}$ does not depend on $x$. We shall also use the 
subspace

$$
\operatorname{ker} R_{x}:=\bigcap_{X, Y \in T_{x} M} \operatorname{ker} R(X, Y) \text {. }
$$

Let us denote by $r$ the type number of $f$ on $M^{\prime}$ and by $\pi$ the projection of $\mathbf{C}^{n+1}$ onto $\mathbf{C}^{r+1}$ parallel to $\mathbf{C}^{n-r} \cong f_{*}(\operatorname{ker} h)$. The following theorem, which in particular gives the full classification of locally symmetric hypersurfaces with $r>2$, has been proved by B. Opozda in [5].

Theorem 1.1. Let $f: M \rightarrow \mathbf{C}^{n+1}$ be a complex hypersurface endowed with a complex transversal vector bundle $\mathcal{N}$ inducing a non-flat locally symmetric connection $\nabla$. Then around every point $x \in M^{\prime}$ there is an open neighbourhood $U$ of $x$ of the form $N^{\prime} \times N^{0}$, where $N^{0}$ endowed with $\nabla$ restricted to $N^{0}$ is affine isomorphic by $f$ to an open subset of $\mathbf{C}^{n-r}$ and $N^{\prime}$ is immersed by $f$ into $\mathbf{C}^{r+1}$ as a non-degenerate hypersurface. If $r>1$, then the bundle $\left.\pi(\mathcal{N})\right|_{U}$ is holomorphic and induces a locally symmetric connection $\nabla^{\prime}$ on $U$ as well as on $N^{\prime}$. If $r>2$, then $\nabla^{\prime}$ is flat or $f\left(N^{\prime}\right)$ is an open part of a central quadric in $\mathbf{C}^{r+1}$. If $r>1$ and $\nabla$ is affine Kähler [i.e., $R(J X, J Y)=R(X, Y)$ for any $X, Y]$, then $\nabla^{\prime}$ is flat.

A local description of complex hypersurfaces with type number one endowed with transversal bundles inducing locally symmetric connections is given in [9].

In the present paper we associate with any locally symmetric complex surface some partial differential equation such that this surface is locally equivalent to the graph of a solution $F$ to this equation and the transversal bundle is also determined by this solution. It is known that the real equation $F_{x x} F_{y y}-F_{x y} F_{x y}=\kappa\left(1+F_{x}{ }^{2}+F_{y}{ }^{2}\right)^{2}$ describes the Euclidean surfaces with constant Gauss curvature $\kappa$. Similar description we obtain for complex locally symmetric surfaces in the case of $\operatorname{dim} \operatorname{im} R=2$, because in this case $\nabla$ turns out to be metrizable in the sense that there exists non-degenerate, C-bilinear, symmetric $g$ such that $\nabla g=0$. The local symmetry implies that the complex sectional curvature of $M$ is then constant. The connection $\nabla$ is induced by the transversal bundle which is perpendicular to $f_{*}(T M)$ with respect to some $\mathbf{C}$-bilinear metric $G$ in $\mathbf{C}^{3}$. The surface $(M, g)$ is isometrically immersed in $\left(\mathbf{C}^{3}, G\right)$.

In the case of $\operatorname{dim} \operatorname{im} R=1$ the equation has the form $F_{z z} F_{w w}-F_{z w} F_{z w}=$ $\Phi\left(F_{z}\right)$ with some arbitrary holomorphic function $\Phi$, which is also associated with the given surface. A local section of the transversal bundle may be expressed in terms of $F_{z}$ and $\eta$, where $\eta$ is a holomorphic function such that 
$\Phi=\frac{\eta}{\eta^{\prime \prime}}$. If $\mathcal{N}$ is the complex affine normal bundle, then $\Phi$ is more strictly determined and the right-hand side of the equation has the form $\left(1+F_{z}{ }^{2}\right)^{2}$.

\section{Locally symmetric connections of rank 1 on surfaces - a class of examples}

Let $V$ be an open subset of $\mathbf{C}$. Let $\eta: V \rightarrow \mathbf{C}$ be a holomorphic function such that $\forall \zeta \in V: \eta(\zeta) \neq 0$ and $\forall \zeta \in V: \eta^{\prime \prime}(\zeta) \neq 0$. Let the holomorphic function $F=F^{1}+i F^{2}: U \rightarrow \mathbf{C}$ of two variables $z=z^{1}+i z^{2}, w=w^{1}+i w^{2}$ satisfy the following partial differential equation:

$$
F_{z z} F_{w w}-F_{z w} F_{z w}=\frac{\eta\left(F_{z}\right)}{\eta^{\prime \prime}\left(F_{z}\right)} .
$$

Let $e_{1}, e_{2}, e_{3}$ be the standard basis of $\mathbf{C}^{3}$. As a local basis of $T M$ over $\mathbf{C}$ we shall use the vector fields $\frac{\partial}{\partial z^{1}}$ and $\frac{\partial}{\partial w^{1}}$. For $\alpha, \beta \in \mathbf{R}$ we have $(\alpha+$ $i \beta) \frac{\partial}{\partial z^{1}}=\alpha \frac{\partial}{\partial z^{1}}+\beta \frac{\partial}{\partial z^{2}}$ and likewise for the $w$-variables.

Proposition 2.1. The transversal vector field

$$
\xi=f_{*}\left(-T_{0}\right)+e_{3}
$$

with

$$
T_{0}=\frac{\eta^{\prime}\left(F_{z}\right)}{\eta\left(F_{z}\right)} \frac{\partial}{\partial z^{1}}
$$

induces on the surface

$$
f: U \ni(z, w) \mapsto(z, w, F(z, w)) \in \mathbf{C}^{3}
$$

a real holomorphic, locally symmetric connection of rank 1 .

Proof. A connection $\nabla$ is real holomorphic if and only if its curvature tensor $R$ satisfies the condition $R(J X, Y)=J R(X, Y)$ for all $X, Y$ [4]. From the Cauchy-Riemann equations for the holomorphic function $\eta^{\prime}\left(F_{z}\right) / \eta\left(F_{z}\right)$ it follows easily that $\nabla_{J Y} T_{0}=J \nabla_{Y} T_{0}$ for any $Y$. Consequently $D_{J Y} \xi=J D_{Y} \xi$ for any $Y$, which is equivalent to the condition that $\xi$ is real holomorphic and $S, \tau$ are $\mathbf{C}$-linear (see [4]). Therefore $R(J X, Y) Z=J R(X, Y) Z$ for any $X$ by the Gauss equation.

Using the Gauss and Weingarten formulae for the immersion (2.4), which we identify with $\left(z^{1}, z^{2}, w^{1}, w^{2}\right) \mapsto\left(z^{1}, z^{2}, w^{1}, w^{2}, F^{1}(z, w), F^{2}(z, w)\right)$, and 
the transversal field (2.2), where $e_{3}$ when looked at as an element of $\mathbf{R}^{6}$ is equal to $(0,0,0,0,1,0)$, we easily obtain

$$
\begin{aligned}
h\left(\frac{\partial}{\partial z^{i}}, \frac{\partial}{\partial z^{j}}\right) & =\frac{\partial^{2} F^{1}}{\partial z^{i} \partial z^{j}}+i \frac{\partial^{2} F^{2}}{\partial z^{i} \partial z^{j}}, \\
h\left(\frac{\partial}{\partial z^{i}}, \frac{\partial}{\partial w^{j}}\right) & =\frac{\partial^{2} F^{1}}{\partial z^{i} \partial w^{j}}+i \frac{\partial^{2} F^{2}}{\partial z^{i} \partial w^{j}}, \\
h\left(\frac{\partial}{\partial w^{i}}, \frac{\partial}{\partial w^{j}}\right) & =\frac{\partial^{2} F^{1}}{\partial w^{i} \partial w^{j}}+i \frac{\partial^{2} F^{2}}{\partial w^{i} \partial w^{j}}, \\
\nabla_{X} \frac{\partial}{\partial z^{1}}=X\left(F_{z}\right) T_{0} & , \quad \nabla_{Y} \frac{\partial}{\partial w^{1}}=Y\left(F_{w}\right) T_{0} . \\
S X & =\nabla_{X} T_{0}, \\
\tau(X) & =-h\left(X, T_{0}\right) .
\end{aligned}
$$

Here for a complex valued function $f=f_{1}+i f_{2}$ by $X(f)$ we mean $X\left(f_{1}\right)+$ $i X\left(f_{2}\right)$ and for a holomorphic function $F$ we have

$$
\frac{\partial F}{\partial z}=\frac{\partial F^{1}}{\partial z^{1}}+i \frac{\partial F^{2}}{\partial z^{1}}, \quad \frac{\partial F}{\partial w}=\frac{\partial F^{1}}{\partial w^{1}}+i \frac{\partial F^{2}}{\partial w^{1}}
$$

Using (2.3), (2.6) and (2.7), we obtain

$$
S X=X\left(F_{z}\right) \frac{\eta^{\prime \prime}\left(F_{z}\right)}{\eta\left(F_{z}\right)} \frac{\partial}{\partial z^{1}}
$$

From (1.7), (2.1) and (2.5), it follows that

$$
\begin{aligned}
R\left(\frac{\partial}{\partial z^{1}}, \frac{\partial}{\partial w^{1}}\right) \frac{\partial}{\partial z^{1}} & =F_{w z} S \frac{\partial}{\partial z^{1}}-F_{z z} S \frac{\partial}{\partial w^{1}} \\
& =\left(F_{w z} F_{z z}-F_{z z} F_{w z}\right) \frac{\eta^{\prime \prime}\left(F_{z}\right)}{\eta\left(F_{z}\right)} \frac{\partial}{\partial z^{1}}=0 \\
R\left(\frac{\partial}{\partial z^{1}}, \frac{\partial}{\partial w^{1}}\right) \frac{\partial}{\partial w^{1}} & =F_{w w} S \frac{\partial}{\partial z^{1}}-F_{z w} S \frac{\partial}{\partial w^{1}} \\
& =\left(F_{w w} F_{z z}-F_{z w} F_{w z}\right) \frac{\eta^{\prime \prime}\left(F_{z}\right)}{\eta\left(F_{z}\right)} \frac{\partial}{\partial z^{1}}=\frac{\partial}{\partial z^{1}},
\end{aligned}
$$

and it is easy to check that $\nabla R=0$. 


\section{The classification theorem}

Here and subsequently, $\mathcal{A}^{\rightarrow}$ denotes the linear part of an affine map $\mathcal{A}$. The symbol dim stands for the complex dimension $\operatorname{dim}_{\mathbf{C}}$.

Theorem 3.1. Let $M$ be a two-dimensional complex manifold and $f: M \rightarrow \mathbf{C}^{3}$ a non-degenerate holomorphic immersion. Assume that $M$ is endowed with $\mathcal{C}^{\infty}$ transversal bundle $\mathcal{N}$ inducing on $M$ a non-flat locally symmetric connection $\nabla$. Let $R$ be the curvature tensor of $\nabla$.

Then for any $m_{0} \in M$ there exist a neighbourhood $U$ of $m_{0}$, a complex chart $\varphi: U \rightarrow \mathbf{C}^{2}$, an affine complex isomorphism $\mathcal{A}$ of $\mathbf{C}^{3}$ and a holomorphic function $F$ of two variables such that

(i) $\mathcal{A} \circ f \circ \varphi^{-1}(z, w)=(z, w, F(z, w))$,

(ii) $\xi=(\mathcal{A} \circ f)_{*}\left(-T_{0}\right)+e_{3}$, with some vector field $T_{0}$ on $U$, is a local section of $\mathcal{A} \rightarrow \mathcal{N}$.

Moreover, $\mathcal{A}$ and $\varphi$ may be chosen in such a way that $F$ and $T_{0}$ satisfy the following conditions:

(iii) If $\operatorname{dim} \operatorname{im} R=1$, then $T_{0}$ is described by (2.3) with some holomorphic function $\eta$ of one variable and $F$ satisfies the differential equation (2.1).

(iv) If $\operatorname{dim} \operatorname{im} R=1$ and $\mathcal{N}$ is the complex affine normal bundle, then

$$
T_{0}=\frac{F_{z}}{1+F_{z}^{2}} \frac{\partial}{\partial z^{1}}
$$

and $F$ satisfies the differential equation

$$
F_{z z} F_{w w}-F_{z w} F_{z w}=\left(1+F_{z}^{2}\right)^{2}
$$

(v) If $\operatorname{dim} \operatorname{im} R=2$, then $\mathcal{N}$ is the complex affine normal bundle,

$$
T_{0}=\frac{F_{z}}{1+F_{z}^{2}+F_{w}{ }^{2}} \frac{\partial}{\partial z^{1}}+\frac{F_{w}}{1+F_{z}^{2}+F_{w}^{2}} \frac{\partial}{\partial w^{1}}
$$

and $F$ satisfies the differential equation:

$$
F_{z z} F_{w w}-F_{z w} F_{z w}=\left(1+F_{z}^{2}+F_{w}^{2}\right)^{2}
$$

Proof. Let $m_{0} \in M$. Since the immersion $f$ is locally a graph, we may choose a complex chart $\varphi_{1}$ on some neighbourhood $U$ of $m_{0}$ and a complex 
isomorphism $\mathcal{A}_{1}$ of $\mathbf{C}^{3}$ such that

$$
\mathcal{A}_{1} \circ f \circ \varphi_{1}^{-1}(z, w)=(z, w, F(z, w))
$$

with a holomorphic function $F$ of two variables $z, w$ and such that

$$
\mathcal{A}_{1}^{\rightarrow} \mathcal{N}_{m_{0}}=\mathbf{C} e_{3}
$$

We may assume that $\left(\frac{\partial}{\partial z^{1}}\right)_{m_{0}} \notin \operatorname{ker} R_{m_{0}}$ and $\left(\frac{\partial}{\partial w^{1}}\right)_{m_{0}} \notin \operatorname{ker} R_{m_{0}}$, for if not, we replace $\varphi_{1}$ by $\psi \circ \varphi_{1}$ and $\mathcal{A}_{1}$ by $\mathcal{A}_{2} \circ \mathcal{A}_{1}$, where $\psi(z, w)=(\alpha z+\beta w, \gamma z+$ $\delta w)$ and $\mathcal{A}_{2}(z, w, u)=(\alpha z+\beta w, \gamma z+\delta w, u)$ with some appropriate complex constants $\alpha, \beta, \gamma, \delta$. We can also assume, by decreasing $U$ if necessary, that the condition $\frac{\partial}{\partial z^{1}} \notin \operatorname{ker} R$ and $\frac{\partial}{\partial w^{1}} \notin \operatorname{ker} R$ is satisfied on the whole of $U$.

The pair $\left(\mathcal{A}_{1} \circ f, \mathcal{A}_{1} \vec{N}\right)$ induces on $M$ the same connection $\nabla$ as the pair $(f, \mathcal{N})$ and $\mathcal{A}_{1} \circ f$ is also a non-degenerate immersion.

Let $\widehat{\xi}: U \rightarrow \mathbf{C}^{3}$ be a local section of $\mathcal{A}_{1} \mathcal{N}$. Since $e_{3}$ is transversal to $\left(\mathcal{A}_{1} \circ f\right)_{*}\left(T_{m_{0}} M\right)$, on some neighbourhood $U^{\prime}$ of $m_{0}$ we have a decomposition $\widehat{\xi}=\left(\mathcal{A}_{1} \circ f\right)_{*}\left(-T_{1}\right)+\lambda e_{3}$ where $\lambda$ is a complex valued function such that $\forall x \in U^{\prime}: \lambda(x) \neq 0$. Dividing $\widehat{\xi}$ by $\lambda$ we obtain the section $\xi=$ $-\left(\mathcal{A}_{1} \circ f\right)_{*}\left(T_{0}\right)+e_{3}$ of $\mathcal{A}_{1} \mathcal{N}$. From (3.6) it follows that $T_{0 m_{0}}=0$. From the Gauss and Weingarten formulae we obtain (2.5) to (2.8) for $\nabla, h, S$ and $\tau$ induced by $\left(\mathcal{A}_{1} \circ f, \xi\right)$.

Locally symmetric connection is semi-symmetric, which means that $R(X, Y) \cdot R=0$ for any $X, Y$; here $R(X, Y)$ acts on $R$ as a derivation. Therefore for any $m \in U$ we can apply to $h_{m}, S_{m}$ and $R_{m}$ the following algebraic lemma [5].

Lemma 01 Let $\mathcal{V}$ be a complex vector space, $\operatorname{dim}_{\mathbf{C}} \mathcal{V}>1$, endowed with a C-bilinear symmetric non-degenerate form $h$. Let $R$ be a tensor of type $(1,3)$ on $\mathcal{V}$ and $S$ an $\mathbf{R}$-linear endomorphism of $\mathcal{V}$ satisfying the Gauss equation

$$
R(X, Y) Z=h(Y, Z) S X-h(X, Z) S Y \text {. }
$$

If for every $X \in \mathcal{V}, R(X, J X) \cdot R=0$, then $S$ is complex [C-linear].

The following two lemmas are consequences of the C-linearity of $S$.

Lemma 3.2. If $\nabla R=0$, then $\nabla$ is a real holomorphic connection, that is, $R(X, Y)$ is $\mathbf{C}$-linear in $X$ and $Y$.

Proof. The claimed C-linearity of $R$ follows from the Gauss equation (1.7). 
Lemma 3.3. $\xi$ is a holomorphic section of $\mathcal{A}_{1} \mathcal{N}$ and $T_{0}$ is a holomorphic vector field.

Proof. From (2.8) and from the C-bilinearity of $h$ it follows that $\tau$ is C-linear. We have now

$$
\begin{aligned}
D_{J X} \xi & =-\left(A_{1} \circ f\right)_{*}(S J X)+\tau(J X) \xi \\
& =-\left(A_{1} \circ f\right)_{*}(J S X)+\tau(X) J \xi=J D_{X} \xi,
\end{aligned}
$$

therefore $\xi$ is holomorphic. From (2.7) we obtain $\nabla_{J X} T_{0}=S J X=J S X=$ $J \nabla_{X} T_{0}$ and the lemma follows.

Lemma 3.4. There exist a neighbourhood $U^{\prime}$ of $m_{0}$ and a holomorphic function $H=A+i B: U^{\prime} \rightarrow \mathbf{C}$ such that $\tau=d A+i d B$.

Proof. We use here a part of another lemma from [5].

Lemma $\mathbf{O} 2$ Let $\mathcal{V}$ be a complex vector space endowed with a $\mathbf{C}$-bilinear symmetric non-degenerate form $h$. Let $R$ be a tensor of type $(1,3)$ on $\mathcal{V}$ and $S$ an $\mathbf{R}$-linear endomorphism of $\mathcal{V}$ satisfying the Gauss equation. If $\operatorname{dim}_{\mathbf{C}} \mathcal{V}>2$, then $R \cdot R=0$ if and only if $S=\lambda \operatorname{id} \mathcal{V}$ for some $\lambda \in \mathbf{C}$. If $\operatorname{dim}_{\mathbf{C}} \mathcal{V}=2$, then $R \cdot R=0$ if and only if $h(X, S Y)=h(S Y, X)$ for every $X, Y \in \mathcal{V}$.

From the Ricci equation (1.10) it follows that $d \tau=0$, which implies $d \mu=0$ and $d \nu=0$ on $U$. Hence there exist a neighbourhood $U^{\prime}$ of $m_{0}$ and real functions $A$ and $B$ on $U^{\prime}$ such that $\mu=d A$ and $\nu=d B$. Since $\tau$ is C-linear (Equation (2.8)), $A+i B$ is holomorphic.

We first consider the case $\operatorname{dim} \operatorname{im} R=1$.

Lemma 3.5. $\operatorname{dim} \operatorname{ker} R=1$.

Proof. We fix a point $x \in U$, where $U$ is the domain of the chart $\varphi$. Let $X_{1}, X_{2}$ be a basis of $T_{x} M$ over $\mathbf{C}$. Since $S J=J S, R$ is $\mathbf{C}$-linear with respect to any variable. Therefore $Z \in \operatorname{ker} R_{x}$ if and only if $R\left(X_{1}, X_{2}\right) Z=0$. Since the type number of the immersion is greater than $1, \operatorname{im} R_{x}=\operatorname{im}_{\mathbf{C}} S_{x}$ [5]. For the complex $S$ we have $\operatorname{im}_{\mathrm{C}} S_{x}=\operatorname{im} S_{x}$. By assumption, $\operatorname{dim} \operatorname{im} R_{x}=1$, therefore $\operatorname{dim} \operatorname{im} S_{x}=1$. Hence $S X_{1}$ and $S X_{2}$ are linearly dependent over C. There exist complex numbers $\alpha, \beta,(\alpha, \beta) \neq(0,0)$, such that $\alpha S X_{1}+$ $\beta S X_{2}=0$. From the non-degeneracy of $h$ it follows that there exists a 
solution $\gamma, \delta$ of the system of linear equations:

$$
\begin{aligned}
& h\left(X_{1}, X_{1}\right) \gamma+h\left(X_{1}, X_{2}\right) \delta=-\beta, \\
& h\left(X_{2}, X_{1}\right) \gamma+h\left(X_{2}, X_{2}\right) \delta=\alpha .
\end{aligned}
$$

Of course $(\gamma, \delta) \neq(0,0)$. Therefore $Z_{0}:=\gamma X_{1}+\delta X_{2}$ is non-zero. Using (1.7) and the system (3.7) it is easy to check that $R\left(X_{1}, X_{2}\right) Z_{0}=0$. Since $\nabla$ is non-flat, $\operatorname{ker} R_{x}=\mathbf{C} Z_{0}$.

Lemma 3.6. Let $Z_{0}$ be a non-zero vector from $\operatorname{ker} R_{x}$. Then for any $X, Y \in$ $T_{x} M, R\left(R\left(X, Z_{0}\right) X, Z_{0}\right) Y=0$.

Proof. Since $\nabla$ is semi-symmetric, we have

$$
\begin{aligned}
0= & \left(R\left(X, Z_{0}\right) \cdot R\right)\left(X, Z_{0}\right) Y \\
= & R\left(X, Z_{0}\right)\left(R\left(X, Z_{0}\right) Y\right)-R\left(R\left(X, Z_{0}\right) X, Z_{0}\right) Y \\
& -R\left(X, R\left(X, Z_{0}\right) Z_{0}\right) Y-R\left(X, Z_{0}\right)\left(R\left(X, Z_{0}\right) Y\right) \\
= & -R\left(R\left(X, Z_{0}\right) X, Z_{0}\right) Y-R\left(X, R\left(X, Z_{0}\right) Z_{0}\right) Y \\
= & -R\left(R\left(X, Z_{0}\right) X, Z_{0}\right) Y .
\end{aligned}
$$

Lemma 3.7. (a) If $R\left(W_{1}, W_{2}\right) Y=0$ for any $Y \in T_{x} M$, then $W_{1}$ and $W_{2}$ are linearly dependent over $\mathbf{C}$.

(b) There exists $X \in T_{x} M$ such that $R\left(X, Z_{0}\right) X \neq 0$.

(c) $\operatorname{im} R_{x}=\operatorname{ker} R_{x}$.

Proof. (a) Suppose, contrary to our claim, that $W_{1}$ and $W_{2}$ are linearly independent over $\mathbf{C}$. Then $T_{x} M$ is generated by $W_{1}$ and $W_{2}$ and $R\left(W_{1}, W_{2}\right) Y=$ 0 implies $Y \in \operatorname{ker} R_{x}$. But this contradicts the assumption of (a), because ker $R_{x}$ is a proper subset of $T_{x} M$.

(b) Suppose the assertion is false. Then $R\left(X, Z_{0}\right) X=0$ and $R\left(X, Z_{0}\right)$ $Z_{0}=0$ for any $X \in T_{x} M$. Using (1.7) we obtain

$$
\begin{gathered}
h\left(Z_{0}, Z_{0}\right) S X-h\left(X, Z_{0}\right) S Z_{0}=0, \\
h\left(Z_{0}, X\right) S X-h(X, X) S Z_{0}=0 .
\end{gathered}
$$

Subtracting (3.9) multiplied by $h\left(X, Z_{0}\right)$ from (3.8) multiplied by $h(X, X)$ yields

$$
\left|\begin{array}{cc}
h\left(Z_{0}, Z_{0}\right) & h\left(Z_{0}, X\right) \\
h\left(X, Z_{0}\right) & h(X, X)
\end{array}\right| S X=0
$$


It follows that if $X$ and $Z_{0}$ are $\mathbf{C}$-linearly independent, then $S X=0$. We choose $Z_{1}$ such that $Z_{0}, Z_{1}$ is a $\mathbf{C}$-basis of $T_{x} M$. By this, $S Z_{1}=0$ and $S\left(Z_{0}+Z_{1}\right)=0$. Consequently, $S Z_{0}=0$ and $S=0$, which contradicts the fact that $\operatorname{dimim} S=\operatorname{dimim} R=1$.

(c) According to (b) we may choose $X_{0} \in T_{x} M$ such that $R\left(X_{0}, Z_{0}\right) X_{0} \neq 0$. By Lemma 3.6 and (a), $R\left(X_{0}, Z_{0}\right) X_{0}$ and $Z_{0}$ are $\mathbf{C}$-linearly dependent. Since $R\left(X_{0}, Z_{0}\right) X_{0} \neq 0$, there exists $\lambda \in \mathbf{C}$ such that $Z_{0}=\lambda R\left(X_{0}, Z_{0}\right) X_{0}$. Hence $Z_{0} \in \operatorname{im} R_{x}$ and $\operatorname{ker} R_{x}=\mathbf{C} Z_{0} \subset \operatorname{im} R_{x}$. By assumption, $\operatorname{dim}_{\mathbf{C}} \operatorname{im} R=1$, and the lemma follows.

\section{Lemma 3.8.}

$$
\nabla_{X}\left(R\left(\frac{\partial}{\partial z^{1}}, \frac{\partial}{\partial w^{1}}\right) T_{0}\right)=h\left(X, T_{0}\right) R\left(\frac{\partial}{\partial z^{1}}, \frac{\partial}{\partial w^{1}}\right) T_{0} .
$$

Proof. From $\nabla R=0$ it follows that

$$
\begin{aligned}
0= & \left(\nabla_{X} R\right)\left(\left(\frac{\partial}{\partial z^{1}}, \frac{\partial}{\partial w^{1}}\right) T_{0}\right) \\
= & \nabla_{X}\left(R\left(\frac{\partial}{\partial z^{1}}, \frac{\partial}{\partial w^{1}}\right) T_{0}\right)-R\left(\nabla_{X} \frac{\partial}{\partial z^{1}}, \frac{\partial}{\partial w^{1}}\right) T_{0} \\
& -R\left(\frac{\partial}{\partial z^{1}}, \nabla_{X} \frac{\partial}{\partial w^{1}}\right) T_{0}-R\left(\frac{\partial}{\partial z^{1}}, \frac{\partial}{\partial w^{1}}\right)\left(\nabla_{X} T_{0}\right) .
\end{aligned}
$$

The last term vanishes, because $\nabla_{X} T_{0}=S X$ and $\operatorname{im} S=\operatorname{im} R=\operatorname{ker} R$ by Lemma 3.7 (c). From Lemma 3.3, it follows that there exist holomorphic functions $\psi_{1}$ and $\psi_{2}$ such that

$$
T_{0}=\psi_{1} \frac{\partial}{\partial z^{1}}+\psi_{2} \frac{\partial}{\partial w^{1}} .
$$

By the C-bilinearity and anti-symmetry of $R(\cdot, \cdot)$, we have from (2.5), (2.6) and (3.12)

$$
\begin{aligned}
R & \left(\nabla_{X} \frac{\partial}{\partial z^{1}}, \frac{\partial}{\partial w^{1}}\right) T_{0}+R\left(\frac{\partial}{\partial z^{1}}, \nabla_{X} \frac{\partial}{\partial w^{1}}\right) T_{0} \\
& =h\left(X, \frac{\partial}{\partial z^{1}}\right) R\left(T_{0}, \frac{\partial}{\partial w^{1}}\right) T_{0}+h\left(X, \frac{\partial}{\partial w^{1}}\right) R\left(\frac{\partial}{\partial z^{1}}, T_{0}\right) T_{0} \\
& =\left(\psi_{1} h\left(X, \frac{\partial}{\partial z^{1}}\right)+\psi_{2} h\left(X, \frac{\partial}{\partial w^{1}}\right)\right) R\left(\frac{\partial}{\partial z^{1}}, \frac{\partial}{\partial w^{1}}\right) T_{0} \\
& =h\left(X, \psi_{1} \frac{\partial}{\partial z^{1}}+\psi_{2} \frac{\partial}{\partial w^{1}}\right) R\left(\frac{\partial}{\partial z^{1}}, \frac{\partial}{\partial w^{1}}\right) T_{0}=h\left(X, T_{0}\right) R\left(\frac{\partial}{\partial z^{1}}, \frac{\partial}{\partial w^{1}}\right) T_{0}
\end{aligned}
$$


Let $U^{\prime}$ and $H$ be as in Lemma 3.4. We may assume that $U^{\prime}$ is connected. From now on we shall write $U$ instead of $U^{\prime}$.

Lemma 3.9. $\nabla_{X}\left(e^{H} R\left(\frac{\partial}{\partial z^{1}}, \frac{\partial}{\partial w^{1}}\right) T_{0}\right)=0$ for any $\left.X \in T M\right|_{U}$.

Proof. It suffices to use (2.8) and Lemma 3.8.

Lemma 3.10. If $\left(T_{0}\right)_{m_{0}} \in \operatorname{ker} R_{m_{0}}$, then $\left(T_{0}\right)_{m} \in \operatorname{ker} R_{m}$ for any $m \in U$.

Proof. Assume that the vector field $W$ on $U$ has the property $\nabla_{X} W=0$ for any $X$. Any two points $x$ and $y$ of $U$ we can connect with some curve $\gamma$. The coordinates of $W_{\gamma(t)}$ in the basis of $T_{\gamma(t)} M$ obtained from a basis of $T_{x} M$ by parallel displacement along $\gamma$ do not depend on $t$. It follows that if $W_{x}=0$ at some $x \in U$, then $W \equiv 0$ on $U$. Now let $W=e^{H} R\left(\frac{\partial}{\partial z^{1}}, \frac{\partial}{\partial w^{1}}\right) T_{0}$. By assumption, $W_{m_{0}}=0$, therefore $W \equiv 0$ and $R\left(\frac{\partial}{\partial z^{1}}, \frac{\partial}{\partial w^{1}}\right) T_{0}=e^{-H} W \equiv 0$.

Lemma 3.11. For any $m \in U, \psi_{1}(m)=0$ if and only if $\psi_{2}(m)=0$.

Proof. To obtain a contradiction, suppose for example that $\psi_{1}(m)=0$ and $\psi_{2}(m) \neq 0$. Then $\left.\frac{\partial}{\partial w^{1}}\right|_{m}=\frac{1}{\psi_{2}(m)} T_{0 m}$, which contradicts the assumption that $\frac{\partial}{\partial w^{1}} \notin \operatorname{ker} R$.

Lemma 3.12. There exists an open dense subset $U_{2}$ of $U$ such that $\psi_{1} \neq 0$ everywhere on $U_{2}$.

Proof. Suppose that $\psi_{1} \equiv 0$ on some open, non-empty subset $V$ of $U$. Then, by Lemma $3.11, \psi_{2} \equiv 0$ on $V$ and consequently $T_{0} \equiv 0$ on $V$. This contradicts the fact that $\operatorname{dim} \operatorname{im} S=1$, because $\nabla_{X} T_{0}=S X$.

Lemma 3.13. There exists a constant $C \neq 0$ such that $\psi_{2}=C \psi_{1}$ on $U$.

Proof. Let $U_{3}$ be a connected, open, non-empty subset of $U_{2}$ and let $X \in$ $\left.T M\right|_{U_{3}}$. From the equality $\operatorname{im} S=\operatorname{im} R=\operatorname{ker} R$ and from $T_{0} \in \operatorname{ker} R$ it follows that $X\left(\psi_{1}\right) \frac{\partial}{\partial z^{1}}+X\left(\psi_{2}\right) \frac{\partial}{\partial w^{1}} \in \operatorname{ker} R$, because

$$
\begin{aligned}
X\left(\psi_{1}\right) \frac{\partial}{\partial z^{1}}+X\left(\psi_{2}\right) \frac{\partial}{\partial w^{1}} & =\nabla_{X} T_{0}-\psi_{1} \nabla_{X} \frac{\partial}{\partial z^{1}}-\psi_{2} \nabla_{X} \frac{\partial}{\partial w^{1}} \\
& =S X-\psi_{1} h\left(X, \frac{\partial}{\partial z^{1}}\right) T_{0}-\psi_{2} h\left(X, \frac{\partial}{\partial w^{1}}\right) T_{0}
\end{aligned}
$$


Since $T_{0} \in \operatorname{ker} R$ and $\operatorname{dim} \operatorname{ker} R=1$, the tangent vectors $\psi_{1} \frac{\partial}{\partial z^{1}}+\psi_{2} \frac{\partial}{\partial w^{1}}$ and $X\left(\psi_{1}\right) \frac{\partial}{\partial z^{1}}+X\left(\psi_{2}\right) \frac{\partial}{\partial w^{1}}$ are linearly dependent over C. Consequently

$$
X\left(\frac{\psi_{2}}{\psi_{1}}\right)=\frac{1}{\left(\psi_{1}\right)^{2}} \cdot\left|\begin{array}{cc}
\psi_{1} & \psi_{2} \\
X\left(\psi_{1}\right) & X\left(\psi_{2}\right)
\end{array}\right|=0 .
$$

It follows that $X\left(\frac{\psi_{2}}{\psi_{1}}\right)=0$ for any $m \in U_{3}$, for any $X \in T_{m} M$. Since $U_{3}$ is connected, there exists a constant $C$ such that $\frac{\psi_{2}}{\psi_{1}}=C$ on $U_{3}$. The constant $C \neq 0$, for if not, then $\psi_{2} \equiv 0$. Now $\psi_{2}-C \psi_{1}$ is a holomorphic function defined on the connected subset $U$ of $M$ and equal to zero on an open, non-empty set $U_{3}$. From the identity principle for holomorphic functions it follows that $\psi_{2}-C \psi_{1} \equiv 0$ on $U$.

Let $(\widetilde{z}, \widetilde{w})=\varphi_{3}(z, w):=(z,-C z+w)$ and $\mathcal{A}_{3}(z, w, u)=(z,-C z+w, u)$. Then $\mathcal{A}_{3} \circ \mathcal{A}_{1} \circ f \circ\left(\varphi_{3} \circ \varphi_{1}\right)^{-1}(\widetilde{z}, \widetilde{w})=\mathcal{A}_{3} \circ \mathcal{A}_{1} \circ f \circ \varphi_{1}^{-1}(\widetilde{z}, C \widetilde{z}+\widetilde{w})=\mathcal{A}_{3}(\widetilde{z}$, $C \widetilde{z}+\widetilde{w}, F(\widetilde{z}, C \widetilde{z}+\widetilde{w}))=(\widetilde{z}, \widetilde{w}, F(\widetilde{z}, C \widetilde{z}+\widetilde{w}))=(\widetilde{z}, \widetilde{w}, \widetilde{F}(\widetilde{z}, \widetilde{w}))$,

$$
\mathcal{A}_{3} \xi=-\left(\mathcal{A}_{3} \circ \mathcal{A}_{1} \circ f\right)_{*}\left(T_{0}\right)+\mathcal{A}_{3} e_{3}=-\left(\mathcal{A}_{3} \circ \mathcal{A}_{1} \circ f\right)_{*}\left(T_{0}\right)+e_{3},
$$

$\frac{\partial}{\partial \widetilde{z}^{1}}=\frac{\partial}{\partial z^{1}}+C \frac{\partial}{\partial w^{1}}$ and $\frac{\partial}{\partial \widetilde{w}^{1}}=\frac{\partial}{\partial w^{1}}$. Using the new coordinates we can rewrite $T_{0}$ as

$$
T_{0}=\psi_{1}\left(\frac{\partial}{\partial z^{1}}+C \frac{\partial}{\partial w^{1}}\right)=\psi_{1} \frac{\partial}{\partial \widetilde{z}^{1}}=\alpha(\widetilde{z}, \widetilde{w}) \frac{\partial}{\partial \widetilde{z}^{1}}
$$

where $\alpha=\psi_{1} \circ \varphi_{1}^{-1} \circ \varphi_{3}^{-1}$. From now on we write $z, w, F$ instead of $\widetilde{z}, \widetilde{w}, \widetilde{F}$. Then the formulae (2.6) to (2.8) hold.

Lemma 3.14. There exist an open neighbourhood $U^{\prime}$ of $m_{0}$ and a holomorphic function $g$ of one variable such that $\alpha(z, w)=g\left(F_{z}(z, w)\right)$ on $\varphi_{3} \circ$ $\varphi_{1}\left(U^{\prime}\right)$.

Proof. An easy computation shows that

$$
\nabla_{X} T_{0}=X\left(\psi_{1}\right) \frac{\partial}{\partial z^{1}}+\psi_{1}^{2} h\left(X, \frac{\partial}{\partial z^{1}}\right) \frac{\partial}{\partial z^{1}}
$$

and

$$
R\left(\frac{\partial}{\partial z^{1}}, \frac{\partial}{\partial w^{1}}\right) \frac{\partial}{\partial z^{1}}=\left(\frac{\partial^{2} F}{\partial w \partial z} \frac{\partial \alpha}{\partial z}-\frac{\partial^{2} F}{\partial z \partial z} \frac{\partial \alpha}{\partial w}\right) \frac{\partial}{\partial z^{1}} .
$$


By Lemma 3.12 and by (3.14) $\alpha \neq 0$ on some dense open subset $\widetilde{U}$ of $\varphi_{3} \circ$ $\varphi_{1}(U)$. For any $(z, w) \in \widetilde{U}$ we can write

$$
\left(\frac{\partial^{2} F}{\partial w \partial z} \frac{\partial \alpha}{\partial z}-\frac{\partial^{2} F}{\partial z \partial z} \frac{\partial \alpha}{\partial w}\right) \frac{\partial}{\partial z^{1}}=R\left(\frac{\partial}{\partial z^{1}}, \frac{\partial}{\partial w^{1}}\right)\left(\frac{1}{\alpha(z, w)} T_{0}\right)=0
$$

Hence

$$
\frac{\partial^{2} F}{\partial w \partial z} \frac{\partial \alpha}{\partial z}-\frac{\partial^{2} F}{\partial z \partial z} \frac{\partial \alpha}{\partial w}=0
$$

on $\widetilde{U}$ and, by continuity, on $\varphi_{3} \circ \varphi_{1}(U)$. Furthermore, $\frac{\partial^{2} F}{\partial z \partial z} \neq 0$ or $\frac{\partial^{2} F}{\partial w \partial z} \neq$ 0 for any point $m \in M$, since otherwise $\frac{\partial}{\partial z^{1}} \in$ ker $h$ which contradicts the non-degeneracy of $f$. Therefore $\Psi:=\frac{\partial F}{\partial z}$ satisfies the assumptions of the following lemma. Applying Lemma 3.15 to $\Psi$ and $\Lambda:=\alpha$ completes the proof of Lemma 3.14.

Lemma 3.15. Let $V$ be an open subset of $\mathbf{C}^{2}$. Let $\Psi: V \rightarrow \mathbf{C}$ be a holomorphic function of two variables such that for any $(z, w) \in V, \frac{\partial \Psi}{\partial z}(z, w) \neq 0$ or $\frac{\partial \Psi}{\partial w}(z, w) \neq 0$. Then $\Lambda: V \rightarrow \mathbf{C}$ satisfies the equation

$$
\frac{\partial \Psi}{\partial z} \frac{\partial \Lambda}{\partial w}-\frac{\partial \Psi}{\partial w} \frac{\partial \Lambda}{\partial z}=0
$$

if and only if for any $\left(z_{0}, w_{0}\right) \in V$ there exist an open neighbourhood $V^{\prime}$ of $\left(z_{0}, w_{0}\right)$ and a holomorphic function $g: \Psi\left(V^{\prime}\right) \rightarrow \mathbf{C}$ of one variable such that $\left.\Lambda\right|_{V^{\prime}}=\left.g \circ \Psi\right|_{V^{\prime}}$.

Proof. is similar to that of constant-rank mapping theorem. Let $\Lambda$ satisfy (3.19). It follows that rank of the holomorphic mapping

$$
V \ni(z, w) \mapsto(\Psi(z, w), \Lambda(z, w)) \in \mathbf{C}^{2}
$$

is equal to 1 on $V$. Let $\left(z_{0}, w_{0}\right) \in V$. Without loss of generality we can assume that $\frac{\partial \Psi}{\partial z}\left(z_{0}, w_{0}\right) \neq 0$. Then there exists a neighbourhood $V^{\prime}$ of $\left(z_{0}, w_{0}\right)$ such that $\Phi: V^{\prime} \ni(z, w) \mapsto(\Psi(z, w), w) \in \Phi\left(V^{\prime}\right) \subset \mathbf{C}^{2}$ is biholomorphic. We may also assume that $\Phi\left(V^{\prime}\right)$ is a product of two open discs $D_{1} \subset \mathbf{C}$ and $D_{2} \subset \mathbf{C}$. Let $\widetilde{g}(u, v):=\Lambda\left(\Phi^{-1}(u, v)\right)$. Rank of the mapping

$$
(\Psi, \Lambda) \circ \Phi^{-1}: \Phi\left(V^{\prime}\right) \ni(u, v) \mapsto(u, \widetilde{g}(u, v)) \in \mathbf{C}^{2}
$$

is also equal to 1 , therefore $\frac{\partial \widetilde{g}}{\partial v}(u, v)=0$ for any $(u, v) \in D_{1} \times D_{2}$. Let $u \in D_{1}$. Since the function $D_{2} \ni v \mapsto \widetilde{g}(u, v) \in \mathbf{C}$ is a constant one, we 
may define $g(u):=\widetilde{g}(u, v)$ with an arbitrary $v \in D_{2}$. We have then $(\Psi, \Lambda) \circ$ $\Phi^{-1}(u, v)=(u, g(u))$. If we take $(u, v)=\Phi(z, w)=(\Psi(z, w), w)$, the assertion follows.

Conversely, let $\Lambda=g \circ \Psi$ on some open set $V^{\prime}$. Applying the chain rule we obtain $\frac{\partial \Lambda}{\partial z}(z, w)=g^{\prime}(\Psi(z, w)) \frac{\partial \Psi}{\partial z}(z, w)$ and $\frac{\partial \Lambda}{\partial w}(z, w)=g^{\prime}(\Psi(z, w)) \frac{\partial \Psi}{\partial w}$ $(z, w)$. Multiplying the first equation by $\frac{\partial \Psi}{\partial w}(z, w)$, the second by $\frac{\partial \Psi}{\partial z}(z, w)$ and subtracting we obtain (3.19).

Let $\left(z_{0}, w_{0}\right)=\varphi_{3} \circ \varphi_{1}\left(m_{0}\right)$. We can decrease the neighbourhood $U^{\prime}$ of $m_{0}$ so as to obtain a connected, simply connected open neighbourhood $F_{z}\left(U^{\prime}\right)$ of $\zeta_{0}:=F_{z}\left(z_{0}, w_{0}\right)$. The holomorphic function $\zeta \mapsto \int_{\gamma\left(\zeta_{0}, \zeta\right)} g(\sigma) d \sigma$, where $\gamma\left(\zeta_{0}, \zeta\right)$ denotes a path joining $\zeta_{0}$ with $\zeta$, is then well defined on $F_{z}\left(U^{\prime}\right)$. Let

$$
\eta(\zeta):=e^{\int_{\gamma\left(\zeta_{0}, \zeta\right)} g(\sigma) d \sigma}
$$

We have then $g(\zeta)=\frac{\eta^{\prime}(\zeta)}{\eta(\zeta)}$ and

$$
S X=\nabla_{X} T_{0}=\frac{\eta^{\prime \prime}\left(F_{z}\right)}{\eta\left(F_{z}\right)} X\left(F_{z}\right) \frac{\partial}{\partial z^{1}} .
$$

Since $\operatorname{dimim} S=1, \eta^{\prime \prime}\left(F_{z}\right) \neq 0$ everywhere on $U^{\prime}$.

Lemma 3.16. F satisfies the differential equation

$$
F_{z z} F_{w w}-F_{z w} F_{z w}=\kappa \frac{\eta\left(F_{z}\right)}{\eta^{\prime \prime}\left(F_{z}\right)}
$$

where $\kappa \in \mathbf{C} \backslash\{0\}$.

Proof. Using the Gauss equation and (3.21) we obtain

$$
R\left(\frac{\partial}{\partial z^{1}}, \frac{\partial}{\partial w^{1}}\right) \frac{\partial}{\partial w^{1}}=\left(F_{z z} F_{w w}-F_{z w} F_{w z}\right) \frac{\eta^{\prime \prime}\left(F_{z}\right)}{\eta\left(F_{z}\right)} \frac{\partial}{\partial z^{1}}=: \Phi \frac{\partial}{\partial z^{1}} .
$$

From $\nabla R=0$ it follows that for any $\left.X \in T M\right|_{U^{\prime}}$

$$
\begin{aligned}
0= & \left(\nabla_{X} R\right)\left(\frac{\partial}{\partial z^{1}}, \frac{\partial}{\partial w^{1}}\right) \frac{\partial}{\partial w^{1}}=\nabla_{X}\left(\Phi \frac{\partial}{\partial z^{1}}\right)-R\left(\nabla_{X} \frac{\partial}{\partial z^{1}}, \frac{\partial}{\partial w^{1}}\right) \frac{\partial}{\partial w^{1}} \\
& -R\left(\frac{\partial}{\partial z^{1}}, \nabla_{X} \frac{\partial}{\partial w^{1}}\right) \frac{\partial}{\partial w^{1}}-R\left(\frac{\partial}{\partial z^{1}}, \frac{\partial}{\partial w^{1}}\right)\left(\nabla_{X} \frac{\partial}{\partial w^{1}}\right) .
\end{aligned}
$$


The last two terms vanish, because $\frac{\partial}{\partial z^{1}}$ and $\nabla_{X} \frac{\partial}{\partial w^{1}}$ are linearly dependent and $\nabla_{X} \frac{\partial}{\partial w^{1}}=X\left(F_{w}\right) T_{0}$ with $T_{0} \in \operatorname{ker} R$. Hence

$$
0=X(\Phi) \frac{\partial}{\partial z^{1}}+\Phi X\left(F_{z}\right) T_{0}-X\left(F_{z}\right) R\left(T_{0}, \frac{\partial}{\partial w^{1}}\right) \frac{\partial}{\partial w^{1}}=X(\Phi) \frac{\partial}{\partial z^{1}}
$$

Since $U^{\prime}$ is connected, $\Phi=\mathrm{const}=: \kappa$. From $\eta^{\prime \prime} \neq 0$ and from the nondegeneracy of $f$ it follows that $\kappa \neq 0$.

Let $\beta$ be a complex number such that $\beta^{2}=\kappa$. Let $(\widetilde{z}, \widetilde{w})=\varphi_{4}(z, w)=$ $(z, \beta w), \mathcal{A}_{4}(z, w, u)=(z, \beta w, u), \varphi=\varphi_{4} \circ \varphi_{3} \circ \varphi_{1}$ and $\mathcal{A}=\mathcal{A}_{4} \circ \mathcal{A}_{3} \circ \mathcal{A}_{1}$. It is easy to check that $\mathcal{A} \circ f \circ \varphi^{-1}(z, w)=(z, w, \widehat{F}(z, w))$ where $\widehat{F}(z, w)=$ $F\left(z, \frac{1}{\beta} w\right)$ satisfies the differential equation (2.1). Since at the corresponding points $\widehat{F}_{\widetilde{z}}=F_{z}$ and $\frac{\partial}{\partial \widetilde{z}^{1}}=\frac{\partial}{\partial z^{1}}$, we have a local section of $\mathcal{A}^{\rightarrow} \mathcal{N}$ as claimed.

Having $\mathcal{A}, \varphi, F, \eta$ and $\xi$ which satisfy (i), (ii) and (iii) of Theorem 3.1, we consider now the particular case when $\mathcal{N}$ is the complex affine normal bundle of the immersion $f$.

Lemma 3.17. $\eta^{3} \cdot \eta^{\prime \prime}=c$ with some $c \in \mathbf{C} \backslash\{0\}$.

Proof. The transversal field

$$
\xi_{e q}=\eta\left(F_{z}\right) \xi=-(\mathcal{A} \circ f)_{*}\left(\eta^{\prime}\left(F_{z}\right) \frac{\partial}{\partial z^{1}}\right)+\eta\left(F_{z}\right) e_{3}
$$

is the equiaffine section of the bundle $\mathcal{A}^{\rightarrow} \mathcal{N}$, which is the complex affine normal bundle for $\mathcal{A} \circ f$. Therefore, there exists a complex number $b$ such that $\widehat{\xi}=b \xi_{e q}$ is the complex affine normal vector field for $\mathcal{A} \circ f$. Let $Z=$ $\frac{1}{b \eta\left(F_{z}\right)} \frac{\partial}{\partial z^{1}}, W=\frac{\partial}{\partial w^{1}}$. We have then ${ }^{\mathrm{c}} \omega\left((\mathcal{A} \circ f)_{*}(Z),(\mathcal{A} \circ f)_{*}(W), \widehat{\xi}\right)=1$. By the definition of the affine normal vector field,

$$
\left|\operatorname{det}\left(\begin{array}{cc}
\widehat{h}(Z, Z) & \widehat{h}(Z, W) \\
\widehat{h}(W, Z) & \widehat{h}(W, W)
\end{array}\right)\right|=1
$$

where $\widehat{h}=\frac{1}{b \eta\left(F_{z}\right)} h$ is the affine fundamental form induced by $\widehat{\xi}$. Using $(2.5)$ we obtain

$$
\left|\left(\frac{1}{b \eta\left(F_{z}\right)}\right)^{4}\left(F_{z z} F_{w w}-F_{z w} F_{z w}\right)\right|=1
$$


which together with (2.1) implies

$$
\left|(\eta(\zeta))^{3} \cdot \eta^{\prime \prime}(\zeta)\right|=\left|\frac{1}{b}\right|^{4}=\mathrm{const}
$$

for $\zeta \in F_{z}\left(U^{\prime}\right)$. According to the maximum principle, if for a holomorphic function $\mathcal{F}: \Omega \rightarrow \mathbf{C}$, where $\Omega \subset \mathbf{C}$ is an open and connected set, the function $|\mathcal{F}|$ has a local maximum at some point of $\Omega$, then $\mathcal{F}$ must be constant on $\Omega$. From (3.27) it follows that $\left|\eta^{3} \cdot \eta^{\prime \prime}\right|$ has a local maximum at any $\zeta \in F_{z}\left(U^{\prime}\right)$. Therefore $\eta^{3} \cdot \eta^{\prime \prime}=$ const.

Lemma 3.18. $\eta(\zeta)=\sqrt{A \zeta^{2}+B \zeta+C}$, where $A \in \mathbf{C} \backslash\{0\}, B, C \in \mathbf{C}$, $A C-\frac{B^{2}}{4}=c$ and $\sqrt{\cdot}$ is some holomorphic branch of the square root defined on some neighbourhood of the non-zero complex number $A \zeta_{0}^{2}+B \zeta_{0}+C$, $\zeta_{0}=F_{z}\left(z_{0}, w_{0}\right)$.

Proof. From $T_{0 m_{0}}=0$ and (2.3) it follows that $\eta^{\prime}\left(\zeta_{0}\right)=0$. Let

$$
E(\zeta):=\left(\eta^{\prime}(\zeta)\right)^{2}+\frac{c}{(\eta(\zeta))^{2}}
$$

for $\zeta \in V$, where $V$ is some sufficiently small, connected neighbourhood of $\zeta_{0}$. Using Lemma 3.17 we obtain $E^{\prime}(\zeta)=\frac{2 \eta^{\prime}(\zeta)}{(\eta(\zeta))^{3}}\left((\eta(\zeta))^{3} \cdot \eta^{\prime \prime}(\zeta)-c\right)=0$, therefore $E(\zeta)=E\left(\zeta_{0}\right)=\frac{c}{\left(\eta\left(\zeta_{0}\right)\right)^{2}}$ for $\zeta \in V$. It follows that

$$
\left(\eta^{\prime}(\zeta)\right)^{2}=\frac{c}{\left(\eta\left(\zeta_{0}\right)\right)^{2}}-\frac{c}{(\eta(\zeta))^{2}} .
$$

We consider now the function $\psi(\zeta):=(\eta(\zeta))^{2}$. Using (3.29) and Lemma 3.17 we obtain

$$
\begin{aligned}
\psi^{\prime \prime}(\zeta) & =2\left(\eta^{\prime}(\zeta)\right)^{2}+2 \eta(\zeta) \cdot \eta^{\prime \prime}(\zeta) \\
& =\frac{2 c}{\left(\eta\left(\zeta_{0}\right)\right)^{2}}-\frac{2 c}{(\eta(\zeta))^{2}}+2 \eta(\zeta) \cdot \eta^{\prime \prime}(\zeta)=\frac{2 c}{\left(\eta\left(\zeta_{0}\right)\right)^{2}}
\end{aligned}
$$

It follows that

$$
\psi(\zeta)=\frac{c}{\left(\eta\left(\zeta_{0}\right)\right)^{2}} \zeta^{2}+B \zeta+C
$$


Since $\eta^{\prime}\left(\zeta_{0}\right)=0$ implies $\psi^{\prime}\left(\zeta_{0}\right)=0$, we have $B=-\frac{2 c}{\left(\eta\left(\zeta_{0}\right)\right)^{2}} \zeta_{0}$. Computing $\psi\left(\zeta_{0}\right)$ we obtain $C=\left(\eta\left(\zeta_{0}\right)\right)^{2}+\frac{c \zeta_{0}^{2}}{\left(\eta\left(\zeta_{0}\right)\right)^{2}}$ and

$$
\psi(\zeta)=\frac{c}{\left(\eta\left(\zeta_{0}\right)\right)^{2}}\left(\zeta-\zeta_{0}\right)^{2}+\left(\eta\left(\zeta_{0}\right)\right)^{2}
$$

Since $\eta\left(\zeta_{0}\right) \neq 0$, there exists a holomorphic branch $\sqrt{\cdot}$ of square root on some neighbourhood of $\left(\eta\left(\zeta_{0}\right)\right)^{2}$. But $\eta$ is also holomorphic, therefore we may conclude, replacing $\sqrt{\cdot}$ by $-\sqrt{\cdot}$ if necessary, that $\eta(\zeta)=\sqrt{\psi(\zeta)}$.

Lemma 3.19. There exist an affine isomorphism $\mathcal{A}_{8}$ of $\mathbf{C}^{3}$ and a local diffeomorphism $\varphi_{8},(\widetilde{z}, \widetilde{w})=\varphi_{8}(z, w)$ such that

$$
\mathcal{A}_{8} \circ \mathcal{A} \circ f \circ \varphi^{-1} \circ \varphi_{8}^{-1}(\widetilde{z}, \widetilde{w})=(\widetilde{z}, \widetilde{w}, \widetilde{F}(\widetilde{z}, \widetilde{w})) .
$$

$\widetilde{F}$ satisfies the differential equation

$$
\widetilde{F}_{\widetilde{z} \widetilde{F}} \widetilde{F}_{\widetilde{w} \widetilde{w}}-\widetilde{F}_{\widetilde{z} \widetilde{F}} \widetilde{F}_{\widetilde{z} \widetilde{w}}=\left(1+\widetilde{F}_{\widetilde{z}}^{2}\right)^{2}
$$

and $-\mathcal{A}_{8} \circ \mathcal{A} \circ f_{*}\left(T_{0}\right)+e_{3}$ with

$$
T_{0}=\frac{\widetilde{F}_{\widetilde{z}}}{1+\widetilde{F}_{\widetilde{z}}^{2}} \frac{\partial}{\partial \widetilde{z}^{1}}
$$

is a local section of $\mathcal{A}_{8} \rightarrow \mathcal{A} \rightarrow \mathcal{N}$.

Proof. For $\eta$ as in Lemma 3.18 we have

$$
\begin{aligned}
\frac{\eta(\zeta)}{\eta^{\prime \prime}(\zeta)}=\frac{1}{c}(\eta(\zeta))^{4}=\frac{A C-\left(B^{2} / 4\right)}{A^{2}} & \\
& {\left[\left(\frac{A}{\sqrt{A C-\left(B^{2} / 4\right)}} \zeta+\frac{(B / 2)}{\sqrt{A C-\left(B^{2} / 4\right)}}\right)^{2}+1\right]^{2} . }
\end{aligned}
$$

Let $\varphi_{8}(z, w)=\left(\frac{\sqrt{A C-\left(B^{2} / 4\right)}}{A} z, w\right), \mathcal{A}_{8}(z, w, u)=\left(\frac{\sqrt{A C-\left(B^{2} / 4\right)}}{A} z, w, u+\frac{B}{2 A} z\right)$ and

$$
\widetilde{F}(\widetilde{z}, \widetilde{w})=F\left(\frac{A}{\sqrt{A C-\left(B^{2} / 4\right)}} \widetilde{z}, \widetilde{w}\right)+\frac{(B / 2)}{\sqrt{A C-\left(B^{2} / 4\right)}} \widetilde{z} .
$$


It is easy to check that at the corresponding points

$$
\frac{A}{\sqrt{A C-\left(B^{2} / 4\right)}} F_{z}+\frac{(B / 2)}{\sqrt{A C-\left(B^{2} / 4\right)}}=\widetilde{F}_{\widetilde{z}}
$$

and

$$
F_{z z} F_{w w}-F_{z w} F_{z w}=\frac{A C-\left(B^{2} / 4\right)}{A^{2}}\left(\widetilde{F}_{\widetilde{z} \widetilde{z}} \widetilde{F}_{\widetilde{w} \widetilde{w}}-\widetilde{F}_{\widetilde{z} \widetilde{w}} \widetilde{F}_{\widetilde{z} \widetilde{w}}\right)
$$

therefore $\widetilde{F}$ satisfies Equation (3.32). Since $\mathcal{A}_{8} e_{3}=e_{3}$, we do not have to change $T_{0}$ but it should be described in the new coordinates. We have at the corresponding points

$$
\begin{aligned}
\frac{\eta^{\prime}\left(F_{z}\right)}{\eta\left(F_{z}\right)} & =\frac{A F_{z}+(B / 2)}{\frac{1}{A}\left(A F_{z}+(B / 2)\right)^{2}+C-\left(B^{2} / 4 A\right)} \\
& =\frac{\widetilde{F}_{\widetilde{z}} \sqrt{A C-\left(B^{2} / 4\right)}}{\frac{A C-\left(B^{2} / 4\right)}{A}\left(\widetilde{F}_{\widetilde{z}}^{2}+1\right)}=\frac{A}{\sqrt{A C-\left(B^{2} / 4\right)}} \frac{\widetilde{F}_{\widetilde{z}}}{\widetilde{F}_{\widetilde{z}}^{2}+1}, \\
\frac{\partial}{\partial z^{1}} & =\frac{\sqrt{A C-\left(B^{2} / 4\right)}}{A} \frac{\partial}{\partial \widetilde{z}^{1}}
\end{aligned}
$$

and the lemma follows.

We now turn to the case $\operatorname{dim} \operatorname{im} R=2$. The shape operator $S$ is then invertible. We first show that there exists a $\mathbf{C}$-bilinear, complex valued nondegenerate symmetric holomorphic tensor field $g$ such that $\nabla g=0$. Let

$$
g(X, Y):=e^{2 H} h\left(S^{-1} X, Y\right)
$$

where $H$ is a holomorphic function as in Lemma 3.4 and $h, S, \tau$ are induced by the pair $\left(\mathcal{A}_{1} \circ f, \xi\right)$, or, equivalently, by $\left(f,\left(\mathcal{A}_{1}\right)^{-1} \xi\right)$ on some neighbourhood of $m_{0}$. Since $H$ is defined up to a constant, we may assume that $H_{m_{0}}=$ 0 . Since $S$ is $\mathbf{C}$-linear and $h \mathbf{C}$-bilinear, $g$ is $\mathbf{C}$-bilinear. It is non-degenerate because $h$ is non-degenerate and $S_{x}$ is an isomorphism at any $x$. According to Lemma O2, $h\left(S^{-1} X, Y\right)=h\left(S^{-1} X, S S^{-1} Y\right)=h\left(S S^{-1} X, S^{-1} Y\right)=$ $h\left(X, S^{-1} Y\right)=h\left(S^{-1} Y, X\right)$, therefore $g$ is symmetric. 
We fix now some basis $Z, W$ of $T_{x} M$ and define $\alpha: T_{x} M \rightarrow T_{x} M$ and $L: T_{x} M \times T_{x} M \rightarrow \mathbf{C}:$

$$
\begin{gathered}
\alpha(Y):=h(W, Y) Z-h(Z, Y) W \\
L(Y, U):=\operatorname{det}\left(\begin{array}{cc}
h(Z, Y) & h(Z, U) \\
h(W, Y) & h(W, U)
\end{array}\right) .
\end{gathered}
$$

Lemma 3.20. (i) $\alpha$ is a C-linear isomorphism.

(ii) $L$ is $\mathbf{C}$-bilinear and anti-symmetric.

(iii) $L(Z, W) \neq 0$.

(iv) $\alpha \circ \alpha=-L(Z, W) \operatorname{id}_{T_{x} M}$.

(v) $h(Y, \alpha(U))=-h(U, \alpha(Y))$ for any $Y, U \in T_{x} M$.

(vi) $L(\alpha(Y), U)=L(Z, W) h(Y, U)$ for any $Y, U \in T_{x} M$.

Proof. (i) and (ii) are obvious, (iii) follows from the non-degeneracy of $h$. To prove (iv) we need only to compute $\alpha \circ \alpha(Z)$ and $\alpha \circ \alpha(W)$. An easy computation shows that $h(Y, \alpha(U))+h(U, \alpha(Y))=0$. For (vi), it suffices to take as $(Y, U)$ the pairs of basis vectors, to use the definition of $\alpha$ and only the anti-symmetry of $L$.

In the following lemmas we will need the assumption that $\nabla R=0$.

Lemma 3.21. For any $X, U$

$$
L(Z, W)\left(\nabla_{X} S\right) U=\left(\nabla_{X} h\right)(W, \alpha(U)) S Z-\left(\nabla_{X} h\right)(Z, \alpha(U)) S W .
$$

Proof. From the Gauss equation (1.7) it follows that

$$
\begin{aligned}
\left(\nabla_{X} R\right)(Z, W) Y= & \left(\nabla_{X} h\right)(W, Y) S Z-\left(\nabla_{X} h\right)(Z, Y) S W \\
& +h(W, Y)\left(\nabla_{X} S\right) Z-h(Z, Y)\left(\nabla_{X} S\right) W .
\end{aligned}
$$

If $\nabla R=0$, then

$$
\begin{gathered}
-\left(\nabla_{X} h\right)(W, Y) S Z+\left(\nabla_{X} h\right)(Z, Y) S W \\
=h(W, Y)\left(\nabla_{X} S\right) Z-h(Z, Y)\left(\nabla_{X} S\right) W=\left(\nabla_{X} S\right)(\alpha(Y)) .
\end{gathered}
$$

We take now $Y=\alpha(U)$ and use Lemma 3.20(iv). 
Lemma 3.22. For any $X, U, Y$

$$
\left(\nabla_{X} h\right)(U, \alpha(Y))-\left(\nabla_{X} h\right)(Y, \alpha(U))=h\left(X, T_{0}\right)[L(U, Y)+h(U, \alpha(Y))] .
$$

Proof. Since both sides are $\mathbf{C}$-bilinear and anti-symmetric with respect to $Y, U$ (see Lemma 3.20(ii) and (v)), it suffices to prove the formula for $U=Z$ and $Y=W$. If we apply Lemma 3.21 to $X=Z, U=W$, next to $X=W$, $U=Z$ and subtract the formulae, then we obtain

$$
\begin{aligned}
L(Z, W)\left[\left(\nabla_{Z} S\right) W-\left(\nabla_{W} S\right) Z\right] \\
\quad=\left[\left(\nabla_{Z} h\right)(W, \alpha(W))-\left(\nabla_{W} h\right)(W, \alpha(Z))\right] S Z \\
-\left[\left(\nabla_{Z} h\right)(Z, \alpha(W))-\left(\nabla_{W} h\right)(Z, \alpha(Z))\right] S W .
\end{aligned}
$$

From the Codazzi equation (1.9) and (2.8) it follows that

$$
\left(\nabla_{Z} S\right) W-\left(\nabla_{W} S\right) Z=h\left(W, T_{0}\right) S Z-h\left(Z, T_{0}\right) S W .
$$

Since $S$ is invertible, $S Z$ and $S W$ are linearly independent over $\mathbf{C}$, therefore

$$
\begin{aligned}
L(Z, W) h\left(W, T_{0}\right) & =\left(\nabla_{Z} h\right)(W, \alpha(W))-\left(\nabla_{W} h\right)(W, \alpha(Z)), \\
L(Z, W) h\left(Z, T_{0}\right) & =\left(\nabla_{Z} h\right)(Z, \alpha(W))-\left(\nabla_{W} h\right)(Z, \alpha(Z)) .
\end{aligned}
$$

Using the Codazzi equation (1.8), (2.8) and Lemma 3.20(v), we obtain

$$
\begin{gathered}
\left(\nabla_{Z} h\right)(W, \alpha(W))=\left(\nabla_{W} h\right)(Z, \alpha(W))-h\left(W, T_{0}\right) h(Z, \alpha(W)), \\
\left(\nabla_{W} h\right)(Z, \alpha(Z))=\left(\nabla_{Z} h\right)(W, \alpha(Z))+h\left(Z, T_{0}\right) h(Z, \alpha(W)) .
\end{gathered}
$$

It follows that

$$
\begin{gathered}
\left(\nabla_{W} h\right)(Z, \alpha(W))-\left(\nabla_{W} h\right)(W, \alpha(Z))=h\left(W, T_{0}\right)[L(Z, W)+h(Z, \alpha(W))], \\
\left(\nabla_{Z} h\right)(Z, \alpha(W))-\left(\nabla_{Z} h\right)(W, \alpha(Z))=h\left(Z, T_{0}\right)[L(Z, W)+h(Z, \alpha(W))] .
\end{gathered}
$$

Since the $\mathbf{C}$-linear mappings

$$
X \mapsto\left(\nabla_{X} h\right)(Z, \alpha(W))-\left(\nabla_{X} h\right)(W, \alpha(Z))
$$

and

$$
X \mapsto h\left(X, T_{0}\right)[L(Z, W)+h(Z, \alpha(W))]
$$

have the same values on the basis vectors $Z, W$, they are equal and the lemma follows. 


\section{Lemma 3.23.}

$$
\left(\nabla_{X} h\right)(\alpha(U), \alpha(Y))=L(Z, W)\left[-\left(\nabla_{X} h\right)(U, Y)+2 h\left(X, T_{0}\right) h(U, Y)\right] .
$$

Proof. We apply Lemma 3.22 to $\alpha(U)$ and $Y$, then we use Lemma 3.20(iv), (v) and (vi).

\section{Lemma 3.24.}

$$
L(Z, W) h\left(\left(\nabla_{X} S\right) U, Y\right)=-\left(\nabla_{X} h\right)(\alpha(S Y), \alpha(U)) .
$$

Proof. Using Lemmas 3.21 and $\mathrm{O} 2$ we obtain

$$
\begin{aligned}
L & (Z, W) h\left(\left(\nabla_{X} S\right) U, Y\right) \\
& =\left(\nabla_{X} h\right)(W, \alpha(U)) h(S Z, Y)-\left(\nabla_{X} h\right)(Z, \alpha(U)) h(S W, Y) \\
& =\left(\nabla_{X} h\right)(W, \alpha(U)) h(Z, S Y)-\left(\nabla_{X} h\right)(Z, \alpha(U)) h(W, S Y) \\
& =\left(\nabla_{X} h\right)(h(Z, S Y) W-h(W, S Y) Z, \alpha(U)) \\
& =-\left(\nabla_{X} h\right)(\alpha(S Y), \alpha(U)) .
\end{aligned}
$$

\section{Lemma 3.25.}

$$
h\left(\left(\nabla_{X} S\right) U, Y\right)=\left(\nabla_{X} h\right)(S Y, U)-2 h\left(X, T_{0}\right) h(S Y, U) .
$$

Proof. From Lemmas 3.23 and 3.24 we have

$$
L(Z, W) h\left(\left(\nabla_{X} S\right) U, Y\right)=L(Z, W)\left[\left(\nabla_{X} h\right)(S Y, U)-2 h\left(X, T_{0}\right) h(S Y, U)\right]
$$

Since $L(Z, W) \neq 0$, the lemma follows.

Lemma 3.26. $\nabla g=0$.

Proof. It suffices to check that $\left(\nabla_{X} g\right)(S U, S Y)=0$ for any $X, U, Y$. We have

$$
\begin{aligned}
& \left(\nabla_{X} g\right)(S U, S Y) \\
& \quad=X(g(S U, S Y))-g\left(\nabla_{X}(S U), S Y\right)-g\left(S U, \nabla_{X}(S Y)\right) \\
& \quad=X\left(e^{2 H} h(U, S Y)\right)-e^{2 H} h\left(\nabla_{X}(S U), Y\right)-e^{2 H} h\left(U, \nabla_{X}(S Y)\right)
\end{aligned}
$$




$$
\begin{aligned}
= & 2 d H(X) e^{2 H} h(U, S Y)+e^{2 H}\left(\nabla_{X} h\right)(U, S Y)+e^{2 H} h\left(\nabla_{X} U, S Y\right) \\
& +e^{2 H} h\left(U, \nabla_{X}(S Y)\right)-e^{2 H} h\left(\nabla_{X}(S U), Y\right)-e^{2 H} h\left(U, \nabla_{X}(S Y)\right) \\
= & -2 h\left(X, T_{0}\right) e^{2 H} h(U, S Y)+e^{2 H}\left(\nabla_{X} h\right)(U, S Y) \\
& +e^{2 H} h\left(S\left(\nabla_{X} U\right), Y\right)-e^{2 H} h\left(\nabla_{X}(S U), Y\right) \\
= & e^{2 H}\left[-2 h\left(X, T_{0}\right) h(U, S Y)+\left(\nabla_{X} h\right)(U, S Y)-h\left(\left(\nabla_{X} S\right) U, Y\right)\right]
\end{aligned}
$$

which is equal to zero by symmetry of $h$ and $\nabla_{X} h$ and by Lemma 3.25.

Let $x \in U$ and let for $X, Y \in T_{x} M$

$$
\begin{gathered}
G_{x}\left(\left(\mathcal{A}_{1} \circ f\right)_{*} X,\left(\mathcal{A}_{1} \circ f\right)_{*} Y\right):=g(X, Y), \\
G_{x}\left(\left(\mathcal{A}_{1} \circ f\right)_{*} X, \xi_{x}\right):=0, \quad G_{x}\left(\xi_{x}, \xi_{x}\right):=e^{2 H} .
\end{gathered}
$$

Lemma 3.27. $D G=0$.

Proof. From $\nabla g=0$ it follows easily that $\left(D_{X} G\right)\left(\left(\mathcal{A}_{1} \circ f\right)_{*} Y,\left(\mathcal{A}_{1} \circ f\right)_{*} U\right)=0$ for any $X, Y, U$. By definition of $g, g(S X, Y)-e^{2 H} h(X, Y)=0$, which implies $\left(D_{X} G\right)\left(\left(\mathcal{A}_{1} \circ f\right)_{*} Y, \xi\right)=0$. Finally, $\left(D_{X} G\right)(\xi, \xi)=0$ because $d H=\tau$.

In that way we have defined a symmetric, C-bilinear mapping $G: \mathbf{C}^{3} \times$ $\mathbf{C}^{3} \rightarrow \mathbf{C}$. It is easy to check that $G$ is non-degenerate.

Remark 3.28. By the formula (3.36) we have defined the metric tensor $g$ only locally. Let $\widetilde{h}, \widetilde{S}$ and $\widetilde{\tau}$ be induced by $\widetilde{\mathcal{A}}_{1} \circ f$ and a local section $\widetilde{\xi}=\left(\widetilde{\mathcal{A}}_{1} \circ f\right)_{*}\left(-\widetilde{T}_{0}\right)+e_{3}$ of $\widetilde{\mathcal{A}}_{1} \mathcal{N}$. Since $\left(\mathcal{A}_{1}\right)^{-1} \xi$ and $\left(\widetilde{\mathcal{A}}_{1}\right)^{-1} \widetilde{\xi}$ are local holomorphic sections of $\mathcal{N}$, there exists a holomorphic function $\phi$ such that $\left(\widetilde{\mathcal{A}}_{1}\right)^{-1} \widetilde{\xi}=\phi\left(\mathcal{A}_{1}\right)^{-1} \xi$ on some neighbourhood $U$ of $m_{0}$. From (1.4), (1.5) and (1.6) we obtain $\widetilde{h}=\frac{1}{\phi} h, \widetilde{S}=\phi S$ and $d \widetilde{H}=d H+d \log \phi$, where log is some holomorphic branch of logarithm in the neighbourhood of $m_{0}$. If $U$ is connected, then we have $\widetilde{H}=H+\log \phi+C, C \in \mathbf{C}$, and $\widetilde{g}=e^{2 C} g$.

Remark 3.29. In [7], B. Opozda has shown that the Ricci tensor Ric of a locally symmetric torsion-free connection of rank 2 on a 2-dimensional real manifold is symmetric and non-degenerate, hence $\nabla$ is the Levi-Civita connection for the metric tensor $g:=$ Ric. Following this, we could in the complex case instead of Ric consider, defined in [5], the complex Ricci tensor $\operatorname{ric}(X, Y)=\frac{1}{2}[\operatorname{Ric}(X, Y)-i \operatorname{Ric}(X, J Y)]$ which for a holomorphic connection $\nabla$ is equal to $\operatorname{tr}_{\mathbf{C}}\{V \mapsto R(V, X) Y\}$. In the case of induced connection 
we obtain $\operatorname{ric}(X, Y)=h(X, Y) \operatorname{tr}_{\mathbf{C}} S-h(S X, Y)$, where $h, S$ are induced by $f$ and some local section of $\mathcal{N}$. The right-hand side does not depend on the particular section, but on $f$ and $\mathcal{N}$ only. From Lemma $\mathrm{O} 2$ it follows that it is symmetric. Let $X \in T_{m} M$ and let $\operatorname{ric}(X, Y)=0$ for any $Y \in T_{m} M$. Then $h\left(\operatorname{tr}_{\mathbf{C}} S X-S X, Y\right)=0$ for any $Y \in T_{m} M$ and from the non-degeneracy of $h$ it follows that $S X=\operatorname{tr}_{\mathbf{C}} S X$, which for 2-dimensional vector space $T_{m} M$ and invertible $S$ implies $X=0$. Therefore ric is non-degenerate. From $\nabla R=0$ and $\nabla J=0$ it follows that $\nabla$ ric $=0$. In this way we can on the whole of $M$ define a $\mathbf{C}$-bilinear metric tensor $\widehat{g}:=$ ric such that $\nabla \widehat{g}=0$. According to the complex version of the Cartan-Norden theorem, there exists a C-bilinear, non-degenerate symmetric $\widehat{G}: \mathbf{C}^{3} \times \mathbf{C}^{3} \rightarrow \mathbf{C}$ such that for $X, Y \in T M$,

$$
\widehat{G}\left(\left(\mathcal{A}_{1} \circ f\right)_{*}(X),\left(\mathcal{A}_{1} \circ f\right)_{*}(Y)\right)=\widehat{g}(X, Y) \quad \text { and } \quad \widehat{G}\left(\left(\mathcal{A}_{1} \circ f\right)_{*}(X), \xi\right)=0
$$

for any local section of $\mathcal{A}_{1} \rightarrow \mathcal{N}$. These conditions together with the nondegeneracy of $\widehat{g}$ are sufficient to prove the following Lemma 3.30, but in Lemma 3.31 we need not only the formula $\widehat{g}(S X, Y)=C_{1} e^{2 H} h(X, Y)$, which may occur in the proof of the Cartan-Norden theorem, or which we may derive using (3.40), but also there should be $C_{1}=1$, because we use (3.36). To this aim we should locally modify $\widehat{g}$.

From $H_{m_{0}}=0$ and $T_{m_{0}}=0$ it follows that $G\left(e_{3}, e_{3}\right)=G\left(\xi_{m_{0}}, \xi_{m_{0}}\right)=$ $e^{2 H_{m_{0}}}=1$. There exists a complex linear isomorphism $\mathcal{A}_{5}$ of $\mathbf{C}^{3}$ such that $\mathcal{A}_{5} e_{1}, \mathcal{A}_{5} e_{2}, \mathcal{A}_{5} e_{3}$ is a $G$-orthonormal basis of $\mathbf{C}^{3}$ and $\mathcal{A}_{5} e_{3}=e_{3}$. Let $\mathcal{A}_{6}:=$ $\mathcal{A}_{5}^{-1}$. We have $\mathcal{A}_{6} e_{3}=\mathcal{A}_{6} \mathcal{A}_{5} e_{3}=e_{3}$. For the given $\mathcal{A}_{5}$ and $\mathcal{A}_{6}$ it is easy to find $\varphi_{6}$ and $\widehat{F}$ such that

$$
\mathcal{A}_{6} \circ \mathcal{A}_{1} \circ f \circ \varphi_{1}^{-1} \circ \varphi_{6}^{-1}(z, w)=(z, w, \widehat{F}(z, w)) .
$$

From $\mathcal{A}_{6} e_{3}=e_{3}$ it follows that $\mathcal{A}_{6} \xi=-\left(\mathcal{A}_{6} \circ \mathcal{A}_{1} \circ f\right)_{*}\left(T_{0}\right)+e_{3}$. We can look at $\nabla, h, S, \tau$ as at objects induced by $\left(\mathcal{A}_{6} \circ \mathcal{A}_{1} \circ f, \mathcal{A}_{6} \xi\right)$. The new function $\widehat{F}$ from now on we shall denote by $F$.

\section{Lemma 3.30.}

$$
T_{0}=\frac{F_{z}}{1+F_{z}^{2}+F_{w}^{2}} \frac{\partial}{\partial z^{1}}+\frac{F_{w}}{1+F_{z}^{2}+F_{w}^{2}} \frac{\partial}{\partial w^{1}} .
$$

Proof. From $(3.41)$ we obtain $\left(\mathcal{A}_{6} \circ \mathcal{A}_{1} \circ f\right)_{*}\left(\frac{\partial}{\partial z^{1}}\right)=e_{1}+F_{z} e_{3},\left(\mathcal{A}_{6} \circ \mathcal{A}_{1} \circ f\right)_{*}$ $\left(\frac{\partial}{\partial w^{1}}\right)=e_{2}+F_{w} e_{3}$, and consequently $\left(\mathcal{A}_{1} \circ f\right)_{*}\left(\frac{\partial}{\partial z^{1}}\right)=\mathcal{A}_{5} e_{1}+F_{z} \mathcal{A}_{5} e_{3}$, 
$\left(\mathcal{A}_{1} \circ f\right)_{*}\left(\frac{\partial}{\partial w^{1}}\right)=\mathcal{A}_{5} e_{2}+F_{w} \mathcal{A}_{5} e_{3}$. Hence

$$
\begin{aligned}
g\left(T_{0}, \frac{\partial}{\partial z^{1}}\right) & =G\left(\xi+\left(\mathcal{A}_{1} \circ f\right)_{*}\left(T_{0}\right),\left(\mathcal{A}_{1} \circ f\right)_{*}\left(\frac{\partial}{\partial z^{1}}\right)\right) \\
& =G\left(\mathcal{A}_{5} e_{3}, \mathcal{A}_{5} e_{1}+F_{z} \mathcal{A}_{5} e_{3}\right)=F_{z} \\
g\left(T_{0}, \frac{\partial}{\partial w^{1}}\right) & =G\left(\xi+\left(\mathcal{A}_{1} \circ f\right)_{*}\left(T_{0}\right),\left(\mathcal{A}_{1} \circ f\right)_{*}\left(\frac{\partial}{\partial w^{1}}\right)\right) \\
& =G\left(\mathcal{A}_{5} e_{3}, \mathcal{A}_{5} e_{2}+F_{w} \mathcal{A}_{5} e_{3}\right)=F_{w} \\
g\left(\frac{\partial}{\partial z^{1}}, \frac{\partial}{\partial z^{1}}\right) & =G\left(\left(\mathcal{A}_{1} \circ f\right)_{*}\left(\frac{\partial}{\partial z^{1}}\right),\left(\mathcal{A}_{1} \circ f\right)_{*}\left(\frac{\partial}{\partial z^{1}}\right)\right) \\
& =G\left(\mathcal{A}_{5} e_{1}+F_{z} \mathcal{A}_{5} e_{3}, \mathcal{A}_{5} e_{1}+F_{z} \mathcal{A}_{5} e_{3}\right)=1+F_{z}^{2} \\
g\left(\frac{\partial}{\partial z^{1}}, \frac{\partial}{\partial w^{1}}\right) & =G\left(\left(\mathcal{A}_{1} \circ f\right)_{*}\left(\frac{\partial}{\partial z^{1}}\right),\left(\mathcal{A}_{1} \circ f\right)_{*}\left(\frac{\partial}{\partial w^{1}}\right)\right) \\
g\left(\frac{\partial}{\partial w^{1}}, \frac{\partial}{\partial w^{1}}\right) & \left.=G\left(\left(\mathcal{A}_{5} e_{1}+F_{z} \mathcal{A}_{5} e_{3}, \mathcal{A}_{5} e_{2}+F_{w} \circ \mathcal{A}_{5} e_{3}\right)=F_{z} F_{w}, \frac{\partial}{\partial w^{1}}\right),\left(\mathcal{A}_{1} \circ f\right)_{*}\left(\frac{\partial}{\partial w^{1}}\right)\right) \\
& =G\left(\mathcal{A}_{5} e_{2}+F_{w} \mathcal{A}_{5} e_{3}, \mathcal{A}_{5} e_{2}+F_{w} \mathcal{A}_{5} e_{3}\right)=1+F_{w}{ }^{2} .
\end{aligned}
$$

Let $T_{0}=a \frac{\partial}{\partial z^{1}}+b \frac{\partial}{\partial w^{1}}$. From (3.42) to (3.46) we obtain the following system of linear equations:

$$
\begin{aligned}
\left(1+F_{z}^{2}\right) a+F_{z} F_{w} b & =F_{z}, \\
F_{z} F_{w} a+\left(1+F_{w}{ }^{2}\right) b & =F_{w} .
\end{aligned}
$$

It remains to find the solution $a, b$ and the lemma follows. We use here the fact that $g$ is non-degenerate, which implies

$$
g\left(\frac{\partial}{\partial z^{1}}, \frac{\partial}{\partial z^{1}}\right) g\left(\frac{\partial}{\partial w^{1}}, \frac{\partial}{\partial w^{1}}\right)-g\left(\frac{\partial}{\partial z^{1}}, \frac{\partial}{\partial w^{1}}\right) g\left(\frac{\partial}{\partial z^{1}}, \frac{\partial}{\partial w^{1}}\right) \neq 0 .
$$

Let $Z, W$ be a basis of $T_{x} M$. For C-bilinear $g$ and a holomorphic connection $\nabla$ such that $\nabla g=0$

$$
\kappa:=\frac{g(R(Z, W) W, Z)}{g(Z, Z) g(W, W)-g(Z, W) g(W, Z)}
$$

is a complex valued analogue of the sectional curvature of 2-dimensional real manifold. It is easy to check that $\kappa$ does not depend on the choice of the basis and depends on $x$ only. 
Lemma 3.31. If $\operatorname{dim}_{\mathbf{C}} M=2, \nabla R=0, g$ is a $\mathbf{C}$-bilinear metric tensor on $U \subset M$ such that $\nabla g=0$ and $U$ is connected, then $\kappa=$ const.

Proof. We take a local basis $E, F$ such that $g(E, E)=g(F, F)=1$ and $g(E, F)=0$. Since $\nabla g=0$, there exists a complex valued 1 -form $\omega$ such that $\nabla_{X} E=\omega(X) F$ and $\nabla_{X} F=-\omega(X) E$. As $\nabla g=0$ and $\nabla R=0$ we have

$$
\begin{aligned}
X(\kappa)= & X(g(R(E, F) F, E)) \\
= & g\left(R\left(\nabla_{X} E, F\right) F+R\left(E, \nabla_{X} F\right) F+R(E, F) \nabla_{X} F, E\right) \\
& +g\left(R(E, F) F, \nabla_{X} E\right)=0
\end{aligned}
$$

because $R(X, Y)=-R(Y, X)$ and $g(R(K, L) M, N)=-g(R(K, L) N, M)$.

Lemma 3.32. F satisfies the differential equation

$$
F_{z z} F_{w w}-F_{z w} F_{z w}=\kappa\left(1+F_{z}^{2}+F_{w}^{2}\right)^{2} .
$$

Proof. Let $H:=-\frac{1}{2} \log \left(1+F_{z}^{2}+F_{w}^{2}\right)$, where log is a holomorphic branch of logarithm, defined in the neighbourhood of 1 . Then

$$
\begin{aligned}
d H(X) & =-\frac{F_{z} X\left(F_{z}\right)+F_{w} X\left(F_{w}\right)}{1+F_{z}{ }^{2}+F_{w}{ }^{2}}=-\frac{F_{z} h\left(X, \partial / \partial z^{1}\right)+F_{w} h\left(X, \partial / \partial w^{1}\right)}{1+F_{z}{ }^{2}+F_{w}{ }^{2}} \\
& =-h\left(X, T_{0}\right)=\tau(X) .
\end{aligned}
$$

Since $T_{0 m_{0}}=0$, from Lemma 3.30 we obtain $F_{z}\left(z_{0}, w_{0}\right)=0$ and $F_{w}\left(z_{0}\right.$, $\left.w_{0}\right)=0$, where $\left(z_{0}, w_{0}\right)=\varphi_{6} \circ \varphi_{1}\left(m_{0}\right)$. Therefore $H_{m_{0}}=0$. It follows that we may use $H$ to define $g$. From (1.7), (2.5) and (3.36), we obtain

$$
g\left(R\left(\frac{\partial}{\partial z^{1}}, \frac{\partial}{\partial w^{1}}\right) \frac{\partial}{\partial w^{1}}, \frac{\partial}{\partial z^{1}}\right)=e^{2 H}\left(F_{z z} F_{w w}-F_{z w} F_{z w}\right)
$$

By (3.44) to (3.46) we have

$$
\begin{aligned}
& g\left(\frac{\partial}{\partial z^{1}}, \frac{\partial}{\partial z^{1}}\right) g\left(\frac{\partial}{\partial w^{1}}, \frac{\partial}{\partial w^{1}}\right)-g\left(\frac{\partial}{\partial z^{1}}, \frac{\partial}{\partial w^{1}}\right) g\left(\frac{\partial}{\partial z^{1}}, \frac{\partial}{\partial w^{1}}\right) \\
& \quad=1+F_{z}{ }^{2}+F_{w}{ }^{2}
\end{aligned}
$$

It follows that $e^{2 H}\left(F_{z z} F_{w w}-F_{z w} F_{z w}\right)=\kappa\left(1+F_{z}{ }^{2}+F_{w}{ }^{2}\right)$, hence

$$
F_{z z} F_{w w}-F_{z w} F_{z w}=\kappa e^{-2 H}\left(1+F_{z}^{2}+F_{w}^{2}\right)=\kappa\left(1+F_{z}^{2}+F_{w}^{2}\right)^{2} .
$$


To prove Lemma 3.32 one can also directly compute $S X$ as $\nabla_{X} T_{0}$, then using the Gauss equation compute $R$ and using (3.44)-(3.46) express $\kappa$ by the derivatives of $F$.

Let $\beta$ be a complex number such that $\beta^{2}=\kappa$. Let $\mathcal{A}_{7}(z, w, u):=$ $(\beta z, \beta w, \beta u), \varphi_{7}(z, w):=(\beta z, \beta w), \mathcal{A}:=\mathcal{A}_{7} \circ \mathcal{A}_{6} \circ \mathcal{A}_{1}$ and $\varphi=\varphi_{7} \circ \varphi_{6} \circ \varphi_{1}$. It is easy to check that $\mathcal{A} \circ f \circ \varphi^{-1}(\widetilde{z}, \widetilde{w})=\left(\widetilde{z}, \widetilde{w}, \beta F\left(\frac{1}{\beta} \widetilde{z}, \frac{1}{\beta} \widetilde{w}\right)\right)$. As a local section of $\mathcal{A} \rightarrow \mathcal{N}$ we take $\frac{1}{\beta} \mathcal{A}_{7} \circ \mathcal{A}_{6} \xi=-(\mathcal{A} \circ f)_{*}\left(\frac{1}{\beta} T_{0}\right)+e_{3}=:-(\mathcal{A} \circ$ $f)_{*}\left(\widetilde{T}_{0}\right)+e_{3}$. Let $\widetilde{F}(\widetilde{z}, \widetilde{w}):=\beta F\left(\frac{1}{\beta} \widetilde{z}, \frac{1}{\beta} \widetilde{w}\right)$, then $\widetilde{F}$ satisfies the differential equation

$$
\widetilde{F}_{\widetilde{z} \widetilde{F}} \widetilde{F}_{\widetilde{w} \widetilde{w}}-\widetilde{F}_{\widetilde{z} \widetilde{w}} \widetilde{F}_{\widetilde{z} \widetilde{w}}=\left(1+\widetilde{F}_{\widetilde{z}}^{2}+\widetilde{F}_{\widetilde{w}}^{2}\right)^{2} .
$$

From $\widetilde{F}_{\widetilde{z}}(\widetilde{z}, \widetilde{w})=F_{z}\left(\frac{1}{\beta} \widetilde{z}, \frac{1}{\beta} \widetilde{w}\right)$ and $\widetilde{F}_{\widetilde{w}}(\widetilde{z}, \widetilde{w})=F_{w}\left(\frac{1}{\beta} \widetilde{z}, \frac{1}{\beta} \widetilde{w}\right)$ it follows that $\widetilde{F}_{\widetilde{z}}(\varphi(m))=F_{z}\left(\varphi_{6} \circ \varphi_{1}(m)\right)$ and $\widetilde{F}_{\widetilde{w}}(\varphi(m))=F_{w}\left(\varphi_{6} \circ \varphi_{1}(m)\right)$, therefore

$$
\begin{aligned}
\widetilde{T}_{0} & =\frac{F_{z}}{1+F_{z}^{2}+F_{w}^{2}} \frac{1}{\beta} \frac{\partial}{\partial z^{1}}+\frac{F_{w}}{1+F_{z}^{2}+F_{w}^{2}} \frac{1}{\beta} \frac{\partial}{\partial w^{1}} \\
& =\frac{\widetilde{F}_{\widetilde{z}}}{1+\widetilde{F}_{\widetilde{z}}^{2}+\widetilde{F}_{\widetilde{w}}^{2}} \frac{\partial}{\partial \widetilde{z}^{1}}+\frac{\widetilde{F}_{\widetilde{w}}}{1+\widetilde{F}_{\widetilde{z}}^{2}+\widetilde{F}_{\widetilde{w}}^{2}} \frac{\partial}{\partial \widetilde{w}^{1}} .
\end{aligned}
$$

It is easy to check that the equiaffine section of $\mathcal{A} \rightarrow \mathcal{N}$

$$
\begin{aligned}
\xi_{\mathrm{eq}}= & -\mathcal{A} \circ f_{*}\left(\frac{F_{z}}{\sqrt{F_{z}^{2}+F_{w}{ }^{2}+1}} \frac{\partial}{\partial z^{1}}+\frac{F_{w}}{\sqrt{F_{z}^{2}+F_{w}^{2}+1}} \frac{\partial}{\partial w^{1}}\right) \\
& +\sqrt{F_{z}^{2}+F_{w}^{2}+1} e_{5}
\end{aligned}
$$

is the complex affine normal vector field.

\section{Examples}

1. Let $4 A C-B^{2}=1, F(z, w)=A z^{2}+B z w+C w^{2}+K z+L w, \eta(\zeta)=$ $e^{\zeta}$. We have then

$$
\begin{gathered}
F_{z z} F_{w w}-F_{z w} F_{z w}=1, \\
T_{0}=\frac{\partial}{\partial z^{1}}, \quad \xi=(-1,0,-2 A z-B w-K+1) .
\end{gathered}
$$

Note that at no point $m_{0}, T_{0 m_{0}}=0$, but in Proposition 2.1 we do not need such point. The equiaffine section of $\mathcal{A N}$ is

$$
\xi_{\text {eq }}=\eta\left(F_{z}\right) \xi=\left(-e^{2 A z+B w+K}, 0, e^{2 A z+B w+K}(-2 A z-B w-K+1)\right) .
$$


2. Let $\quad 4 A C-B^{2}=-1, \quad F(z, w)=A z^{2}+B z w+C w^{2}+K z+L w$, $\eta(\zeta)=\sin \zeta$. Then

$$
\begin{gathered}
F_{z z} F_{w w}-F_{z w} F_{z w}=-1, \\
T_{0}=\cot (2 A z+B w+K) \frac{\partial}{\partial z^{1}}, \\
\xi=(-\cot (2 A z+B w+K), 0,-(2 A z+B w+K) \cot (2 A z+B w+K)+1), \\
\xi_{\text {eq }}=(-\cos (2 A z+B w+K), 0, \\
-(2 A z+B w+K) \cos (2 A z+B w+K)+\sin (2 A z+B w+K)) .
\end{gathered}
$$

3. Let $F(z, w)=z^{2} e^{i w}, \eta(\zeta)=\frac{1}{2} \zeta^{2}$. Then $F_{z z} F_{w w}-F_{z w} F_{z w}=\frac{1}{2} F_{z}^{2}$,

$$
T_{0}=\frac{1}{z} e^{-i w} \frac{\partial}{\partial z^{1}}, \xi=\left(-\frac{1}{z} e^{-i w}, 0,-1\right), \xi_{\text {eq }}=\left(-2 z e^{i w}, 0,-2 z^{2} e^{2 i w}\right)
$$

4. Warped helicoid. An example of locally symmetric complex surface with $\nabla$ induced by the complex affine normal vector field is a warped helicoid (see [2]). Under a suitable parametrization it can be described by a solution $F$ of the differential equation $F_{z z} F_{w w}-F_{z w} F_{z w}=(1+$ $\left.F_{z}{ }^{2}\right)^{2}$ which we obtain, taking in $(2.1) \eta(\zeta)=\sqrt{\zeta^{2}+1}$. In this case the solution is $F(z, w)=\left(z-f_{1}(i w)\right) \tan (i w)+f_{2}(i w)$, where $f_{1}$ and $f_{2}$ are holomorphic functions of one variable. The surface $(z, w) \rightarrow$ $(z, w, F(z, w))$ is a warped helicoid, because $z_{1}:=z, z_{2}:=F(z, w)$, $z_{3}:=i w$ satisfy the equation $\left(z_{1}-f_{1}\left(z_{3}\right)\right) \sin z_{3}=\left(z_{2}-f_{2}\left(z_{3}\right)\right)$ $\cos z_{3}$, and $(z, w, F(z, w))=\mathcal{B}\left(z_{1}, z_{2}, z_{3}\right)$ where $\mathcal{B}:=\left(\begin{array}{ccc}1 & 0 & 0 \\ 0 & 0 & -i \\ 0 & 1 & 0\end{array}\right)$ is an equiaffine transformation. We obtain $T_{0}=\sin (i w) \cos (i w) \frac{\partial}{\partial z^{1}}, \xi=$ $\left(-\sin (i w) \cos (i w), 0, \cos ^{2}(i w)\right)$ and $\xi_{\text {eq }}=(-\sin (i w), 0, \cos (i w))$.

5. As an example of locally symmetric complex surface of rank 2 let us take $F(z, w)=\sqrt{1-z^{2}-w^{2}}$. Then $T_{0}=-\sqrt{1-z^{2}-w^{2}}$ $\left(z \frac{\partial}{\partial z^{1}}+w \frac{\partial}{\partial w^{1}}\right), \quad \xi=\sqrt{1-z^{2}-w^{2}}\left(z, w, \sqrt{1-z^{2}-w^{2}}\right)$ and $\xi_{\text {eq }}=$ $\left(z, w, \sqrt{1-z^{2}-w^{2}}\right)=(z, w, F(z, w))$.

\section{Acknowledgement}

This research was supported by KBN grant no. 1P03A 03426. 


\section{References}

[1] F. Dillen, L. Vrancken and L. Verstraelen, Complex affine differential geometry, Preprint, Reihe Mathematik, Fachbereich 3 T.U. Berlin, No. 153 (1986).

[2] F. Dillen, Locally symmetric complex affine surfaces, J. Geom. 33 (1988), 27-38.

[3] S. Kobayashi and K. Nomizu, Foundations of differential geometry, vol. I, John Wiley and Sons, New York, 1963.

[4] K. Nomizu and T. Sasaki, Affine differential geometry, Cambridge University Press, 1994.

[5] B. Opozda, On some properties of the curvature and Ricci tensors in complex affine geometry, Geom. Dedicata 55 (1995), 141-163.

[6] B. Opozda, Equivalence theorems for complex affine hypersurfaces, Result. Math. 27 (1995), 316-327.

[7] B. Opozda, Some relations between Riemannian and affine geometry, Geom. Dedicata 47 (1993), 225-236.

[8] B. Opozda, Locally symmetric connections on surfaces, Result. Math. 20 (1991), 725-743.

[9] M. Robaszewska, Locally symmetric connections on complex hypersurfaces with type number 1, Geom. Dedicata 114 (2005), 13-47.

InSTYTUT MATEMATYKi,

Akademia Pedagogiczna,

UL. PODCHORȦ̧̇YCH 2,

30-084 Kraków,

POLAND.

E-mail address: robaszew@ap.krakow.pl

Received June 20, 2007 
